\title{
Analysis of Galileo E5 and E5ab code tracking
}

\author{
Youssef Tawk - Cyril Botteron • Aleksandar Jovanovic • \\ Pierre-André Farine
}

Received: 25 November 2010/Accepted: 4 May 2011/Published online: 20 May 2011

(C) Springer-Verlag 2011

\begin{abstract}
The world of global navigation satellite systems has been enhanced with several new or improved signals in space aiming to optimize accuracy, reliability, navigation solution, and interoperability between different constellations. However, such developments bring various challenges to the receivers' designers. For example, acquisition and tracking stages turn into more complex processes while handling the increasing bandwidth requires additional processing power. In this context, we study the code tracking of Galileo E5ab in a full band or of only one of its components, i.e., either E5a or E5b. More specifically, an architecture for tracking the E5 pilot channel as an AltBOC $(15,10)$ or BPSK(10) modulation is introduced, and the performance of well-known discriminator types is analyzed using analytical derivations and simulations of linearity and stability regions, thermal noise tracking errors, multipath error envelopes and tracking thresholds. Different parameters, such as the front-end filter bandwidth, the early/late chip spacing, un-normalized and normalized discriminators, are taken into consideration. The results obtained are used to illustrate the main advantages and drawbacks of tracking the E5 signal as well as to help defining the main tracking loop parameters for an enhanced performance.
\end{abstract}

Keywords Galileo E5 - AltBOC - Code tracking · Stability $\cdot$ Linearity $\cdot$ Sensitivity

Y. Tawk $(\square) \cdot$ C. Botteron - A. Jovanovic · P.-A. Farine Institute of Microengineering (IMT) Electronics and Signal Processing Laboratory, Ecole Polytechnique Fédérale de Lausanne, Breguet 2, 2000 Neuchâtel, Switzerland e-mail: youssef.tawk@epfl.ch

\section{Introduction}

The European Galileo system will use the E5 band to transmit one of the widest signals in the GNSS spectrum using an Alternative Binary Offset Carrier [AltBOC $(15,10)]$ modulation. With the introduction of this signal, the design of Galileo compatible receivers is made even more challenging and complex (Gerein 2005). In fact, the $\operatorname{AltBOC}(15,10)$ is one of the most advanced and promising GNSS signals. It is characterized by a very wide bandwidth, a sharp autocorrelation function, and four complex channels modulated by four different pseudorandom-noise (PRN) codes. The four PRN's allow transmission of the four channels E5a-I, E5a-Q, E5b-I, and E5b-Q. E5a-I and E5b-I are called data channels, since they carry navigation data, whereas E5a-Q and E5b-Q are called pilot channels since they are not data modulated. All these characteristics make the post-processing of this signal very challenging and complicated.

Several papers already discussed the AltBOC modulation and the advantages of tracking the Galileo E5 signal. For example, in Sleewaegen et al. (2004), the authors presented the principles of tracking and processing the AltBOC $(15,10)$ without discussing in details the main features that characterize the tracking loop parameters. In Margaria et al. (2007), the authors proposed an innovative solution for navigation message demodulation for AltBOC receivers, but they did not study the performance of the tracking stage especially the delay locked loop (DLL) part in terms of robustness under different conditions. In Shivaramaiah et al. (2009), the authors exploited the possibilities of tracking this signal in different schemes (e.g. side-band translation, Full band-independent correlation, Look-up table) and proposed a new method to track E5 in full band along with wiping off the data bits on E5a 
and E5b to take advantage of the maximum power possible. In this work also, the part on the DLL performance was missing, as the new proposed algorithm was not tested under different discriminator types or correlator spacings. Moreover, in Shivaramaiah (2009), the author introduced a method to mitigate the code phase multipath by exploiting the frequency diversity used in Galileo E5. In this work too, the code tracking was only partially tackled (the author provided analytical expressions for some discriminators outputs) and the analysis concentrated more on the part of mitigating the code multipath error. Finally, we note that there are also many other papers that explain the structure of the E5 signal and discuss the main advantages of the constant envelope AltBOC(15,10) modulation (Lestarquit et al. 2008; Shivaramaiah and Dempster 2009).

In summary, the literature is still missing a deeper analysis on the tracking stage of the Galileo E5 signal, revealing more precisely the limitations and drawbacks that can be encountered in a practical implementation. In particular, studies on the code tracking robustness and the parameters that affect the performance of the DLL are still lacking. Therefore, our aim in this paper is to unveil more in details the tracking stability and linearity of this signal tracked as an AltBOC $(15,10)$ or $\operatorname{BPSK}(10)$ modulation and to characterize the error sources (code noise, multipath) that affect the code phase estimation and tracking sensitivity. The receiver parameters such as the front-end filter, discriminator type, normalization technique, and correlators spacing are taken into consideration in order to provide additional insights into the robustness of this signal, stressing both its advantages and limitations.

In 'Galileo E5 signal structure' section, we present the structure and characteristics of the E5 signal and show how it can be approximated in a simpler form due to filtering effects and power sharing. We also show the possibility to track this signal in two different modes, as an AltBOC $(15,10)$ or a BPSK(10) modulation, and study the main effects of the proposed approximations. In 'Autocorrelation function' section, the influence of the front-end filtering is considered together with its effects on the amplitude and shape of the autocorrelation function. In 'Delay locked loop' section, we consider the DLL, and in 'DLL discriminators' section, we study the tracking robustness by analyzing the performance of well-known traditional code loop discriminators in terms of stability and linearity under different conditions (i.e. chip spacing, front-end filter bandwidth, and normalization). In 'Code tracking errors' section, the main error sources (i.e. thermal noise and multipath) on the code tracking loops are discussed and derived. In 'Tracking sensitivity' section, the tracking sensitivity is studied and tracking thresholds are derived. Finally, summary and conclusions are drawn in 'Summary and conclusions' section.

\section{Galileo E5 signal structure}

The Galileo E5 signal is Right Hand Circular Polarized (RHCP) and uses an Alternate Binary Offset Carrier AltBOC $(15,10)$ modulation. The carrier, at E5, is modulated by four quasi-orthogonal Pseudo-Random Noise (PRN) codes $\left(c_{\text {E5a-I }}, c_{\text {E5a-Q }}, c_{\text {E5b-I }}\right.$, and $\left.c_{\text {E5a-Q }}\right)$, two navigation messages $\left(d_{\mathrm{E} 5 \mathrm{a}-\mathrm{I}}, d_{\mathrm{E} 5 \mathrm{~b}-\mathrm{I}}\right)$, and one side-band sub-carrier with a rate of $f_{\mathrm{sc}}=15.345 \mathrm{MHz}$. The expressions for the band pass transmitted signal, assuming one satellite, and its base-band representation are given in (1) and (2) (European Space Agency, European Commission 2010):

$S_{\mathrm{E} 5_{t}}(t)=A \mathbf{R}\left[s_{\mathrm{E} 5}(t) \mathrm{e}^{j 2 \pi f_{\mathrm{c}} t}\right]$

$s_{\mathrm{E} 5}(t)=s_{\mathrm{E} 5-\mathrm{I}}(t)+j s_{\mathrm{E} 5-\mathrm{Q}}(t)$

where $A$ is the signal amplitude, and $f_{\mathrm{c}}$ is the carrier frequency equal to $1,191.795 \mathrm{MHz}$. $\mathbf{R}$ denotes the real function. A detailed description of the generation of the Galileo AltBOC-modulated signal $s_{\mathrm{E} 5}(t)$ can be found in European Space Agency, European Commission (2010). The analytical baseband complex envelope representation of $s_{\mathrm{E} 5}(t)$ is given by:

$$
\begin{aligned}
s_{\mathrm{E} 5}(t)= & \frac{1}{2 \sqrt{2}}\left(e_{\mathrm{E} 5 \mathrm{a}-\mathrm{I}}(t)+j e_{\mathrm{E} 5 \mathrm{a}-\mathrm{Q}}(t)\right)\left[\operatorname{sc}_{\mathrm{S}}(t)-j \operatorname{sc}_{\mathrm{S}}\left(t-\frac{T_{\mathrm{sc}}}{4}\right)\right] \\
& +\frac{1}{2 \sqrt{2}}\left(e_{\mathrm{E} 5 \mathrm{~b}-\mathrm{I}}(t)+j e_{\mathrm{E} 5 \mathrm{~b}-\mathrm{Q}}(t)\right)\left[\operatorname{sc}_{\mathrm{S}}(t)+j \operatorname{sc}_{\mathrm{S}}\left(t-\frac{T_{\mathrm{sc}}}{4}\right)\right] \\
& +\frac{1}{2 \sqrt{2}}\left(\bar{e}_{\mathrm{E} 5 \mathrm{a}-\mathrm{I}}(t)+j \bar{e}_{\mathrm{E} 5 \mathrm{a}-\mathrm{Q}}(t)\right)\left[\operatorname{sc}_{\mathrm{P}}(t)-j \operatorname{sc}_{\mathrm{P}}\left(t-\frac{T_{\mathrm{sc}}}{4}\right)\right] \\
& +\frac{1}{2 \sqrt{2}}\left(\bar{e}_{\mathrm{E} 5 \mathrm{~b}-\mathrm{I}}(t)+j \bar{e}_{\mathrm{E} 5 \mathrm{~b}-\mathrm{Q}}(t)\right)\left[\operatorname{sc}_{\mathrm{P}}(t)+j \operatorname{sc}_{\mathrm{P}}\left(t-\frac{T_{\mathrm{sc}}}{4}\right)\right]
\end{aligned}
$$

where the signal components $e_{\mathrm{E} 5 \mathrm{a}-\mathrm{I}}, e_{\mathrm{E} 5 \mathrm{a}-\mathrm{Q}}, e_{\mathrm{E} 5 \mathrm{~b}-\mathrm{I}}$, and $e_{\mathrm{E} 5 \mathrm{~b}-\mathrm{Q}}$ contain the PRN codes and the navigation messages. The respective dashed signal components $\bar{e}_{\mathrm{E} 5 \mathrm{a}-\mathrm{I}}, \bar{e}_{\mathrm{E} 5 \mathrm{a}-\mathrm{Q}}, \bar{e}_{\mathrm{E} 5 \mathrm{~b}-\mathrm{I}}$ and $\bar{e}_{\mathrm{E} 5 \mathrm{~b}-\mathrm{Q}}$ represent the product signals. The symbols $\mathrm{sc}_{\mathrm{S}}$ and $\mathrm{Sc}_{\mathrm{P}}$ represent the four-valued sub-carrier functions for the single signals side bands (SSB) and the product signals side bands (PSB), in which expressions can be found in European Space Agency, European Commission (2010). The existence of the sub-carrier signal splits the spectrum into two symmetric side lobes E5a and E5b centered at $\pm f_{\text {sc }}$ $\mathrm{MHz}$ from the carrier frequency. Its envelope is given by (Avila-Rodriguez 2008):

$G_{\mathrm{AltBOC}}(f)=\frac{4 f_{\mathrm{p}}}{\pi^{2} f^{2}} \frac{\cos ^{2}\left(\frac{\pi f}{f_{\mathrm{p}}}\right)}{a^{2}}\left[a^{2}-a-2 a \cos \left(\frac{\pi f}{4 f_{\mathrm{sc}}}\right)+2\right]$

where $f_{\mathrm{p}}$ is the PRN code frequency equal to $10.23 \mathrm{MHz}$, and $a$ is defined as: 
$a=\cos \left(\frac{\pi f}{2 f_{\mathrm{sc}}}\right)$

The power spectral density of the E5 AltBOC $(15,10)$ signal is one of the widest spectrums in GNSS bands. From the satellite, the signal is transmitted over a $90-\mathrm{MHz}$ bandwidth, and the minimum required bandwidth to receive it is $51.15 \mathrm{MHz}$. The specified minimum received power using a $0 \mathrm{dBi}$ antenna is $-152 \mathrm{dBW}$ (European Space Agency, European Commission 2010) divided equally between the four channels. The difference between the theoretical, i.e. Eq. 3, and transmitted signal generated from a Spirent GSS8000 simulator (Spirent 2008) is shown in Fig. 1. The transmitted signal shown is a snapshot from a spectrum analyzer with a resolution bandwidth of $3 \mathrm{MHz}$ and LogPower average type with an Average/Hold Number of 100 points. The effect of signal filtering beyond $45 \mathrm{MHz}$ from the center frequency is well noticeable.

The role of the product signals is to obtain a constant envelope-modulated signal. The first harmonic of the PSB occurs at $-3 f_{\mathrm{sc}}(-45 \mathrm{MHz})$ and contains $61.5 \%$ of the total power specified for the product sub-carriers, which is $14.64 \%$ of the total E5 power. Hence, the harmonics at $\pm 3 f_{\text {sc }}$ will only contain $0.615 \times 0.1464=$ $9 \%$ of the total power. For the SSB, the first harmonic occurs at $+f_{\mathrm{sc}}(15 \mathrm{MHz})$ from the center frequency and contains $94.96 \%$ of the total power specified for the single sub-carriers, which is $85.36 \%$ of the total E5 power (Lestarquit et al. 2008). Consequently, the main two lobes of the AltBOC signal (E5a and E5b) will contain $0.9496 \times 0.8536=81 \%$ of the total power. As a result, the product signals that do not carry any useful information have very small amplitude and with a frontend bandwidth smaller than $90 \mathrm{MHz}$, most of their power

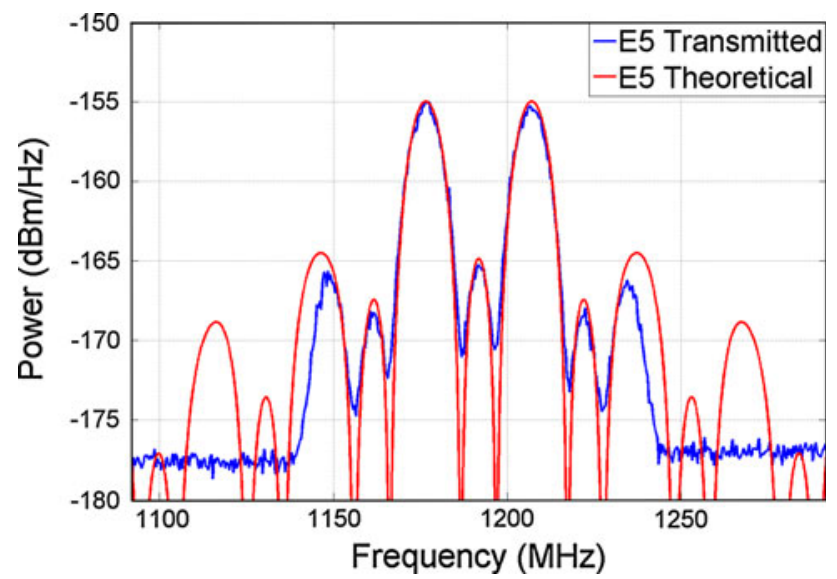

Fig. 1 Power spectral density of the $\operatorname{AltBOC}(15,10)$ and the transmitted Galileo E5 is filtered out. We therefore propose to neglect and rewrite (3) as:

$$
\begin{aligned}
s_{\mathrm{E} 5}(t) \simeq & \frac{1}{2 \sqrt{2}}\left(e_{\mathrm{E} 5 \mathrm{a}-\mathrm{I}}(t)+j e_{\mathrm{E} 5 \mathrm{a}-\mathrm{Q}}(t)\right)\left[\operatorname{sc}_{\mathrm{S}}(t)-j \operatorname{sc}_{\mathrm{S}}\left(t-\frac{T_{\mathrm{sc}}}{4}\right)\right] \\
& +\frac{1}{2 \sqrt{2}}\left(e_{\mathrm{E} 5 \mathrm{~b}-\mathrm{I}}(t)+j e_{\mathrm{E} 5 \mathrm{~b}-\mathrm{Q}}(t)\right)\left[\operatorname{sc}_{\mathrm{S}}(t)+j \operatorname{sc}_{\mathrm{S}}\left(t-\frac{T_{\mathrm{sc}}}{4}\right)\right]
\end{aligned}
$$

Similarly, with a band pass filter smaller than $90 \mathrm{MHz}$, the intermodulation products at $\pm 3 f_{\text {sc }}$ and $\pm 5 f_{\text {sc }}$ are mostly filtered out. Therefore, we also approximate the single subcarrier expressions as pure cosine and sine functions:

$\operatorname{sc}_{\mathrm{S}}(t) \simeq \cos \left(2 \pi f_{\mathrm{sc}} t\right)$

$\operatorname{sc}_{\mathrm{S}}\left(t-\frac{T_{\mathrm{sc}, \mathrm{E} 5}}{4}\right)(t) \simeq \sin \left(2 \pi f_{\mathrm{sc}} t\right)$

Equation 6 can then be written as:

$s_{\mathrm{E} 5}(t) \simeq \frac{1}{2 \sqrt{2}}\left[\mathrm{E} 5 \mathrm{a}(t) e^{-j 2 \pi f_{\mathrm{sc}} t}+\mathrm{E} 5 \mathrm{~b}(t) e^{j 2 \pi f_{\mathrm{sc}} t}\right]$

where $\mathrm{E} 5 \mathrm{a}(t)$ and $\mathrm{E} 5 \mathrm{~b}(t)$ are equal to:

$\mathrm{E} 5 \mathrm{a}(t)=e_{\mathrm{E} 5 \mathrm{a}-\mathrm{I}}(t)+j e_{\mathrm{E} 5 \mathrm{a}-\mathrm{Q}}(t)$

$\mathrm{E} 5 \mathrm{~b}(t)=e_{\mathrm{E} 5 \mathrm{~b}-\mathrm{I}}(t)+j e_{\mathrm{E} 5 \mathrm{~b}-\mathrm{Q}}(t)$

The difference between the autocorrelation function with and without the proposed approximations is shown in Fig. 2. It can be seen that the overall shape of the autocorrelation function is not affected. The only difference is a small amplitude loss at the main peak due to neglecting the power of the PSB that is not filtered out.

Using the above approximations, and taking into account the propagation time, the Galileo E5 signal,

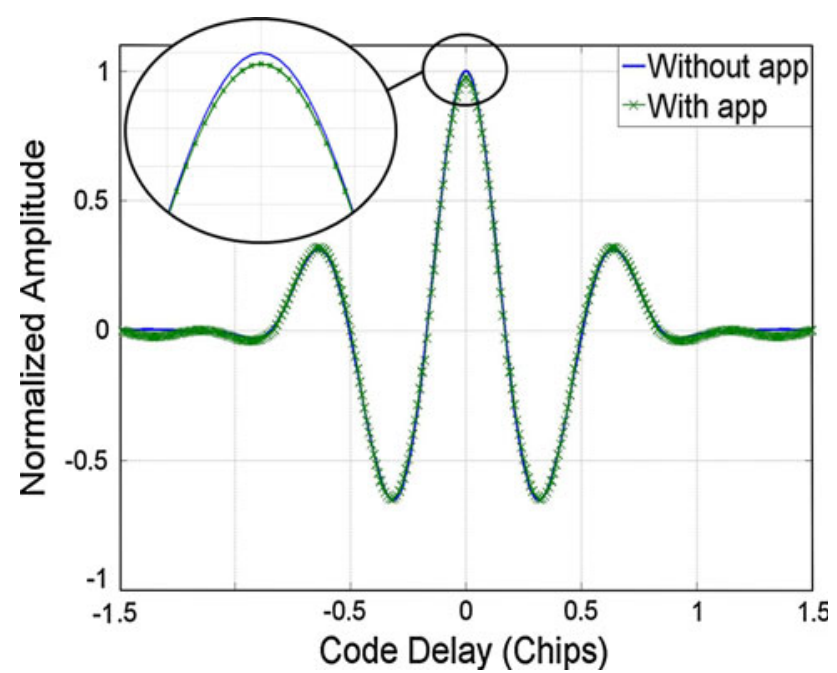

Fig. 2 Comparison of the autocorrelation function for the Galileo E5 with and without the proposed approximations 
coming from a particular satellite, can be modeled at the output of the receiver front end after down conversion and filtering as:

$S_{\mathrm{E} 5_{r}}(t) \simeq A \mathbf{R}\left[\tilde{s}_{\mathrm{E} 5}(t-\tau) \mathrm{e}^{j\left(2 \pi f_{\mathrm{FF}} t+\phi\right)}\right]$

where $\tilde{s}$ is the filtered baseband received signal, $f_{\mathrm{IF}}$ is the intermediate frequency, $\tau$ is the time-varying code delay, and $\phi$ is the time-varying carrier phase delay due to the propagation time. We assume that the phase delay includes the Doppler effect caused by satellite and signal motions. Due to the split spectrum of the E5 signal, it is possible to receive the signal as a pure BPSK $(10)$ modulation when using a filter with a pass-band bandwidth less than $51.15 \mathrm{MHz}$ centered on one of the main peaks corresponding to the E5a or E5b components. In fact, if only one of these two bands is received, then the signal at the output of the front end can be modeled as:

$S_{\mathrm{E} 5 y_{r}}(t)=A \mathbf{R}\left[\tilde{s}_{\mathrm{E} 5 y}(t-\tau) \mathrm{e}^{j\left(2 \pi\left(f_{\mathrm{IF}} \pm f_{\mathrm{sc}}\right) t+\phi\right)}\right]$

where $y$ stands for ' $\mathrm{a}$ ' or ' $\mathrm{b}$ ' depending if $\mathrm{E} 5 \mathrm{a}\left(f_{\mathrm{IF}}-f_{\mathrm{sc}}\right)$ or $\mathrm{E} 5 \mathrm{~b}\left(f_{\mathrm{IF}}+f_{\mathrm{sc}}\right)$ is acquired. Equation 13 clearly results in receiving a $\operatorname{BPSK}(10)$ modulation signal, where the value ' 10 ' stands for a signal that has a PRN code rate 10 times the GPS C/A code rate (i.e. 10.23 MHz). Figure 3 shows the PSD of the E5a signal component generated from a Spirent GSS8000 simulator (Spirent 2008).

In the following, the focus will be on studying the Galileo E5 tracking in full band as an $\operatorname{AltBOC}(15,10)$ modulation or in a limited bandwidth centered on E5a or E5b as a BPSK(10) modulation. But, first, a brief description of the $\operatorname{AltBOC}(15,10)$ autocorrelation function is presented, and the impact of the receiver front-end filtering is discussed.

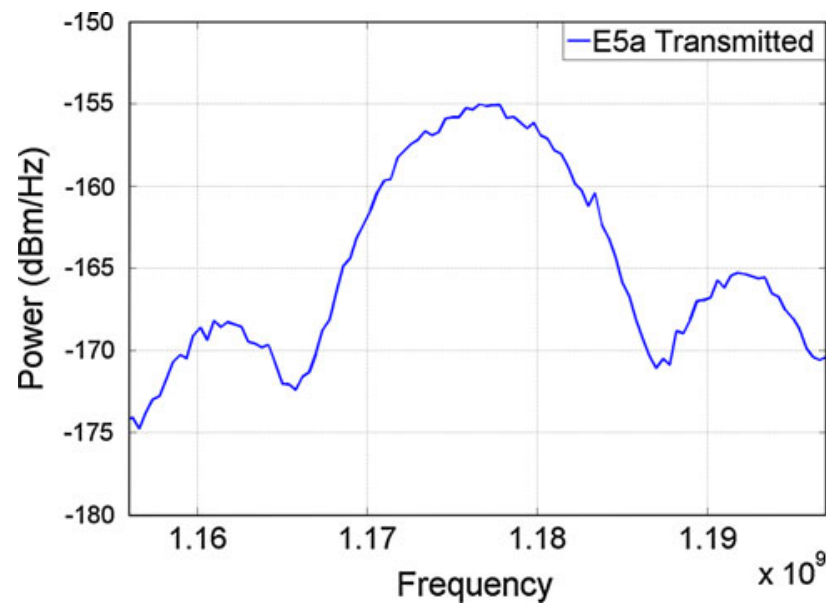

Fig. 3 Power spectral density of the Galileo E5a BPSK(10) for the minimum received power of $-155 \mathrm{dBW}$ (same averaging as in Fig. 1)

\section{Autocorrelation function}

The analysis of the autocorrelation function is carried on the two pilot channels E5a-Q and E5b-Q. As they do not carry navigation data bits, a long integration time can be used and thus a more robust tracking can be obtained. The data channel case will still be addressed shortly at the end of this section. The pilot channel on the E5 signal is obtained by taking only the imaginary part of (10) and (11). At baseband, it can be written as:

$$
\begin{aligned}
s_{Q}(t)= & \tilde{c}_{\mathrm{E} 5 \mathrm{a}-\mathrm{Q}}(t-\tau) \mathrm{e}^{-j\left(2 \pi f_{\mathrm{sc}}(t-\tau)\right)} \\
& +\tilde{c}_{\mathrm{E} 5 \mathrm{~b}-\mathrm{Q}}(t-\tau) \mathrm{e}^{j\left(2 \pi f_{\mathrm{sc}}(t-\tau)\right)}
\end{aligned}
$$

To track the pilot component, $s_{Q}(t)$ should be correlated with locally generated spreading codes $c_{\mathrm{E} 5 \mathrm{a}-\mathrm{Q}}$ and $c_{\mathrm{E} 5 \mathrm{~b}-\mathrm{Q}}$ multiplied by the complex conjugate of the corresponding sub-carrier exponential. The correlation output will then be equal to:

$\tilde{R}_{Q}(\tau)=\tilde{R}_{\mathrm{E} 5 \mathrm{aQ}}(\tau)+\tilde{R}_{\mathrm{E} 5 \mathrm{bQ}}(\tau)$

where $\tilde{R}_{\mathrm{E} 5 \mathrm{aQ}}(\tau)$ and $\tilde{R}_{\mathrm{E} 5 \mathrm{bQ}}(\tau)$ can be calculated as:

$$
\begin{aligned}
\tilde{R}_{\mathrm{E} 5 \mathrm{aQ}}(\tau)= & \int_{T_{\mathrm{int}}} \tilde{c}_{\mathrm{E} 5 \mathrm{a}-\mathrm{Q}}(t-\tau) \mathrm{e}^{-j\left(2 \pi f_{\mathrm{sc}}(t-\tau)\right)} c_{\mathrm{E} 5 \mathrm{a}-\mathrm{Q}}(t) \mathrm{e}^{j\left(2 \pi f_{\mathrm{sc}}(t)\right)} \\
& \approx \tilde{R}(\tau) \mathrm{e}^{-j 2 \pi f_{\mathrm{sc}} \tau} \\
\tilde{R}_{\mathrm{E} 5 \mathrm{bQ}}(\tau)= & \int_{T_{\text {int }}} \tilde{c}_{\mathrm{E} 5 \mathrm{~b}-\mathrm{Q}}(t-\tau) \mathrm{e}^{j\left(2 \pi f_{\mathrm{sc}}(t-\tau)\right)} c_{\mathrm{E} 5 \mathrm{~b}-\mathrm{Q}}(t) e^{-j\left(2 \pi f_{\mathrm{sc}}(t)\right)} \\
& \approx \tilde{R}(\tau) \mathrm{e}^{j 2 \pi f_{\mathrm{sc}} \tau}
\end{aligned}
$$

where $\tilde{R}(\tau)$ is the triangular function, and $T_{\mathrm{int}}$ is the integration time. Replacing (16) and (17) in (15), we can write the autocorrelation function of the Galileo pilot channel as:

$\tilde{R}_{Q}(\tau)=\tilde{R}(\tau) \cos \left(2 \pi f_{\mathrm{sc}} \tau\right)$

Figure 4 shows the autocorrelation function of the E5 pilot signal for different receiver bandwidths along with the theoretical BPSK(10) autocorrelation function. It can be seen that it possesses five peaks in the region of $[-1,+1]$ chip. The effect of receiver filtering on the shape of the autocorrelation function is not significant, but the power at the main peak is reduced. Note that in the case of infinite bandwidth, the product signals are taken into account, but for limited bandwidths, they are neglected as explained before.

A zoom-in around the main peak is shown in Fig. 5, where it can be seen that the peak of the E5 pilot signal is much sharper than that of the BPSK(10). As expected, reducing the front-end filter reduces the amplitude of the main peak and rounds it. Table 1 shows the amplitude losses due to the different front-end filter bandwidths considered. 


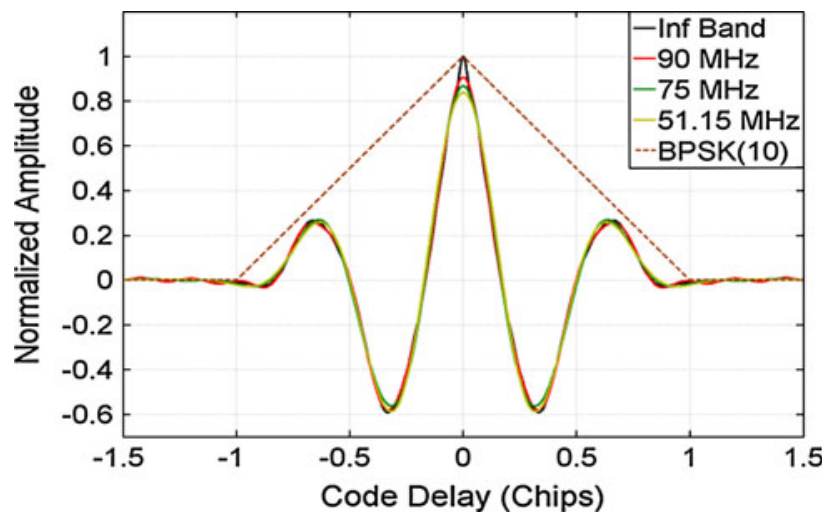

Fig. 4 Impact of the front-end receiver bandwidth on the E5 autocorrelation function

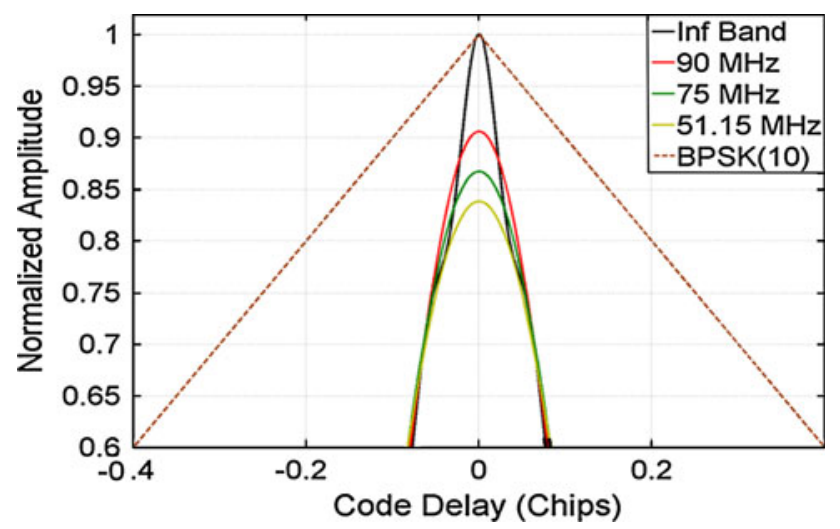

Fig. 5 Zoom-in around the main peak of Fig. 4

In the case of the data channel, and as shown before for the pilot channel, the combined E5a-Q/E5b-Q correlation functions are simply the sum of the individual E5a and E5b pilot correlation functions. For the data channel, the same principle can be used, but the integration time should be less than one data bit navigation unless the data bits are wiped off prior to the combination. The E5-data correlation peak is given by:

$\tilde{R}_{I}(\tau)=\tilde{R}(\tau) \cos \left(2 \pi f_{\mathrm{sc}} \tau\right)$

Analysis on the E5a or E5b autocorrelation function will not be discussed here, as it is a pure BPSK modulation that has been already addressed in the literature for the GPS C/A signal (Kaplan and Hegarty 2006; Betz and Kolodziejski 2000; Braasch and Dierendonck 1999). Concluding this section, Table 2 summarizes the main characteristics of the E5 and E5a or E5b autocorrelation functions.
Table 1 E5 amplitude losses due to receiver front-end filtering

\begin{tabular}{lllr}
\hline Bandwidth (MHz) & 90 & 75 & 51.15 \\
Loss (dB) & 0.42 & 0.61 & 0.76 \\
\hline
\end{tabular}

Table 2 E5 and E5a/E5b characteristics

\begin{tabular}{lll}
\hline Modulation & $\begin{array}{l}\text { E5 } \\
\text { AltBOC }(15,10)\end{array}$ & $\begin{array}{l}\text { E5a or E5b } \\
\text { BPSK(10) }\end{array}$ \\
\hline Min. bandwidth & $51.15 \mathrm{MHz}$ & $20 \mathrm{MHz}$ \\
Main peak width & $\pm 0.33 \mathrm{chip}$ & $\pm 1 \mathrm{chip}$ \\
Min. received power & $-152 \mathrm{dBW}$ & $-155 \mathrm{dBW}$ \\
\hline
\end{tabular}

\section{Delay locked loop (DLL)}

In a conventional GNSS receiver architecture, both a carrier and a PRN code tracking loop are implemented to refine the frequency and code phase rough estimates from the acquisition block, and to keep track of these values and demodulate the navigation data bits from a specific satellite. The carrier wave signal is often tracked using phase lock loop (PLL) and/or frequency lock loop (FLL). The code tracking loop is often a delay lock loop (DLL) called an early/late tracking loop. Code delay tracking is extremely important in a GNSS receiver since it provides the pseudorange measurements and prompt code phases for the PLL. Consequently, in the design of a DLL, it is necessary to have robust, reliable, and accurate measurements. The general architecture of the E5 pilot tracking is shown in Fig. 6 and is similar for E5 data tracking.

The first step in the tracking stage is to wipe-off the carrier by multiplying the incoming signal with a perfectly aligned local carrier replica. Afterward, the resulting in-phase and quadrature-phase components are multiplied with three final code replicas [Early (E), Prompt (P), and Late (L)]. The three replicas are generated according to (16) and (17) with a correlation spacing of $\pm d / 2$. After this second multiplication, the resulting outputs are filtered with the integration and dump filters (I\&D). The output of these integrations is a numerical value indicating how much the specific code replica correlates with the code in the incoming signal. The total combined duration of the receiver and processor integrate and dump functions establishes the pre-detection integration time for the signal $\left(T_{\text {int }}\right)$. Assuming a limited phase and limited frequency variation during $T_{\mathrm{int}}$, the six in-phase and quadrature-phase correlation results can be modeled as:

$$
\begin{gathered}
I_{Y}=\frac{A}{2} \tilde{R}_{Q}\left(\tau-d_{Y}\right) \frac{\sin \left(\pi f_{\mathrm{d}} T_{\mathrm{int}}\right)}{\pi f_{\mathrm{d}} T_{\mathrm{int}}} \cos \left(\epsilon_{\phi}\right)+\eta_{I_{Y}} \\
Q_{Y}=\frac{A}{2} \tilde{R}_{Q}\left(\tau-d_{Y}\right) \frac{\sin \left(\pi f_{\mathrm{d}} T_{\mathrm{int}}\right)}{\pi f_{\mathrm{d}} T_{\mathrm{int}}} \sin \left(\epsilon_{\phi}\right)+\eta_{Q_{Y}}
\end{gathered}
$$


Fig. 6 General tracking architecture of the Galileo E5 pilot channel

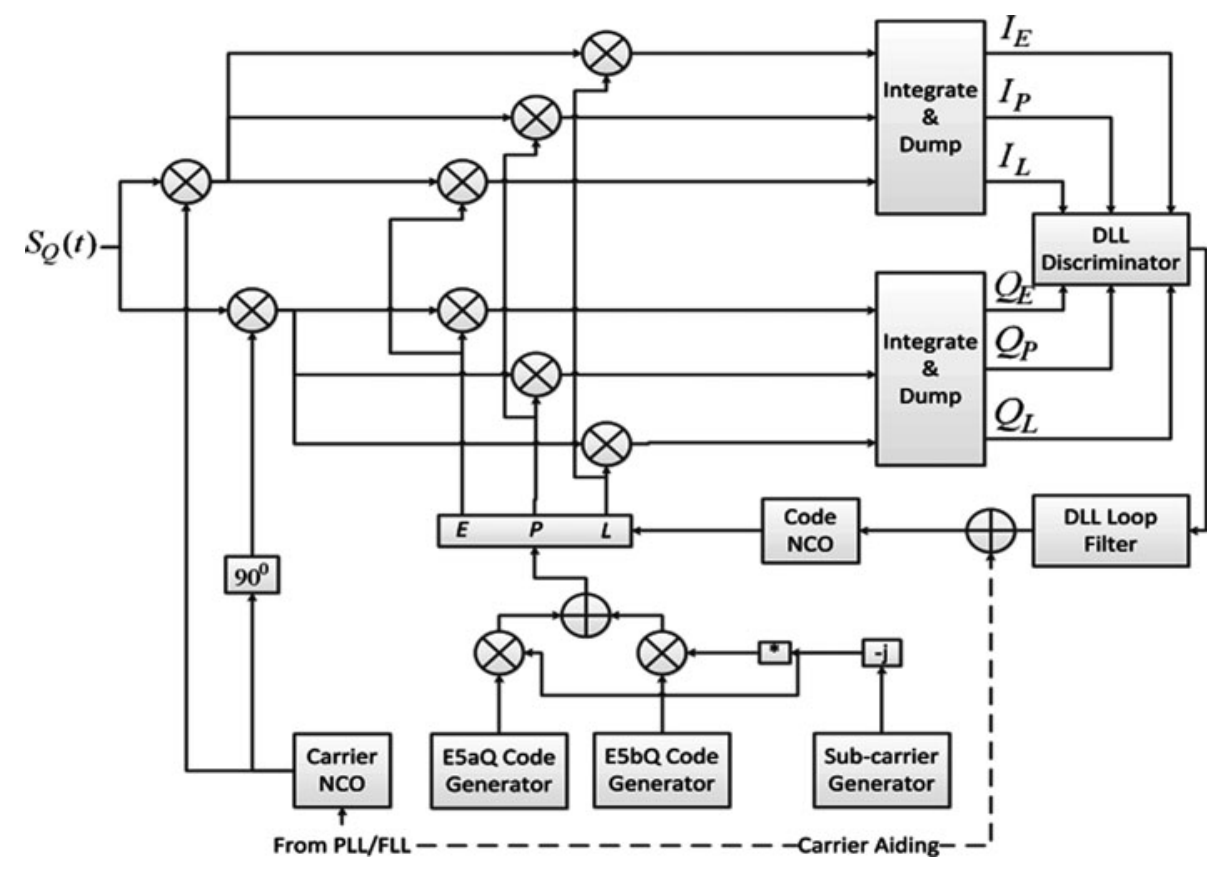

where $Y$ stands for the Early, Prompt, or Late replicas, $d_{Y}$ corresponds to their respective delays [i.e. Early $\left(d_{\mathrm{E}}=\right.$ $+d / 2)$, Prompt $\left(d_{\mathrm{P}}=0\right)$ and Late $\left.\left(d_{\mathrm{L}}=-\mathrm{d} / 2\right)\right], \eta_{I_{Y}}$ and $\eta_{Q_{Y}}$ are independent Gaussian noises, $f_{\mathrm{d}}$ is the Doppler frequency, and $\epsilon_{\phi}$ is the carrier phase error. It is important to mention that these correlation values are dependent on the phase of the local carrier wave. If the local carrier wave is in phase with the received signal, all the energy will be in the in-phase component. But if the local carrier phase drifts compared with the input signal, the energy will switch between the in-phase and the quadrature components. After (I\&D), $I_{Y}$ and $Q_{Y}$ are fed into a DLL discriminator to estimate the code delay error $\tau$. The resulting value is then filtered by a DLL filter, and the output is then fed to the code NCO that synthesizes the Early, Prompt, and Late replica codes. It is important to note that the local code rate and the local carrier frequency Doppler are linked since they are both due to the satellite-receiver signal propagation time variation. However, the resulting difference in the code and carrier Doppler is often minimal, and, consequently, it is very common to use carrier tracking information to aid the code tracking loop (Julien 2005). As frequency estimation from the PLL is usually very accurate, it means that it absorbs the main dynamic component and, consequently, the DLL is not required to perform this task independently. Thus, the DLL loop filter is generally a second-order filter with a narrow bandwidth, as it should then be used mainly to remove the noise.

In the case of the Galileo E5a (or E5b) tracking, the general architecture is similar to Fig. 6, but instead of generating a final code that is a multiplex of E5a and E5b
PRN codes and a sub-carrier, the code generator will only generates the E5a (or E5b) PRN codes without a sub-carrier, and the carrier frequency will be centered on the E5a (or E5b) center frequency equal to $1,173.45 \mathrm{MHz}$ (or 1,207.14 MHz).

The receiver code tracking loop performance will depend on the selected pre-detection integrations, the code loop discriminator, and the code loop filter. While the main characteristics of the loop filter and code NCO can be found in Kaplan and Hegarty (2006), we provide in the following section a more detailed performance analysis for the discriminator considering the $\operatorname{AltBOC}(15,10)$ and BPSK(10) modulations.

\section{DLL discriminators}

The main task of the discriminator is to estimate the code delay error between the incoming and the locally generated spreading codes. Different types of discriminators exist in a GNSS receiver, mainly categorized as coherent or noncoherent discriminator. The most used coherent discriminator type is the early minus late (EML) and is given by:

$D_{\mathrm{EML}}=I_{\mathrm{E}}-I_{\mathrm{L}}$

This discriminator is characterized by its simplicity and linearized response. Its main disadvantage is that it requires a good carrier tracking loop for optimal functionality, and it is sensitive to carrier phase errors. In fact, this highprecision DLL mode fails if there are frequent cycle slips or total loss of phase lock because the phasor rotates, causing the signal power to be shared in both the I and Q 
components, which consequently causes power loss in the coherent DLL. For these reasons, it is not recommended for robust DLL tracking and non-coherent discriminators are often used as they are insensitive to carrier phase errors.

Two types of non-coherent discriminators are widely used: the non-coherent early minus late power (NEMLP) and the Dot-Product (DP). They are given by (Kaplan and Hegarty 2006):

$D_{\mathrm{NEMLP}}=\left(I_{\mathrm{E}}^{2}-I_{\mathrm{L}}^{2}\right)-\left(Q_{\mathrm{E}}^{2}-Q_{\mathrm{L}}^{2}\right)$

$D_{\mathrm{DP}}=\left(I_{\mathrm{E}}-I_{\mathrm{L}}\right) I_{\mathrm{P}}+\left(Q_{\mathrm{E}}-Q_{\mathrm{L}}\right) Q_{\mathrm{P}}$

The NEMLP discriminator has a high computational load as it uses three correlators (E, L, and P), while the DP discriminator has lower computational load as it uses only two correlators (E-L and $\mathrm{P}$ ). It is important to note that these DLL discriminators can be normalized. In fact, normalization removes the amplitude sensitivity, which improves performance under rapidly changing SNR conditions and provides unbiased code delay error estimation. First, analysis considering unnormalized discriminators is conducted, and the effect of normalization is addressed later on.

\section{Unnormalized DLL}

Using (20) and (21) assuming a perfect carrier lock, Eqs. 23 and 24 can be written as:

$D_{\mathrm{NEMLP}}=\frac{A^{2}}{4}\left(\tilde{R}_{Q}^{2}(\tau-d / 2)-\tilde{R}_{Q}^{2}(\tau+d / 2)\right)$

$D_{\mathrm{DP}}=\frac{A^{2}}{4}\left(\tilde{R}_{Q}(\tau-d / 2)-\tilde{R}_{Q}(\tau+d / 2)\right) \tilde{R}_{Q}(\tau)$

To assess the performance and robustness of these two discriminators, two criteria are used: stability and linearity. The stability region is defined as the region surrounding the zero phase error where a certain phase error input will result in a mean discriminator response having the same sign as the input error. This means that, for a certain input error, the discriminator will react in the correct direction and should converge toward zero phase error. The linear tracking region is defined as the region around the zero phase error where a certain phase error input will result in a mean discriminator response equal to the input error. This means that, for a certain input error, the discriminator will react perfectly (that is, without bias) (Julien 2005). Figure 7 shows the stability and linearity region for a typical discriminator output. After studying these two points, the main sources of error (i.e. thermal noise, multipath, dynamic stress) will be discussed.

First, let us start by showing the impact of the front-end filter on the discriminator output. Figures 8 and 9 show the discriminator outputs for a 0.1 chip spacing for the E5a and E5 pilot signals for different front-end filter bandwidths.

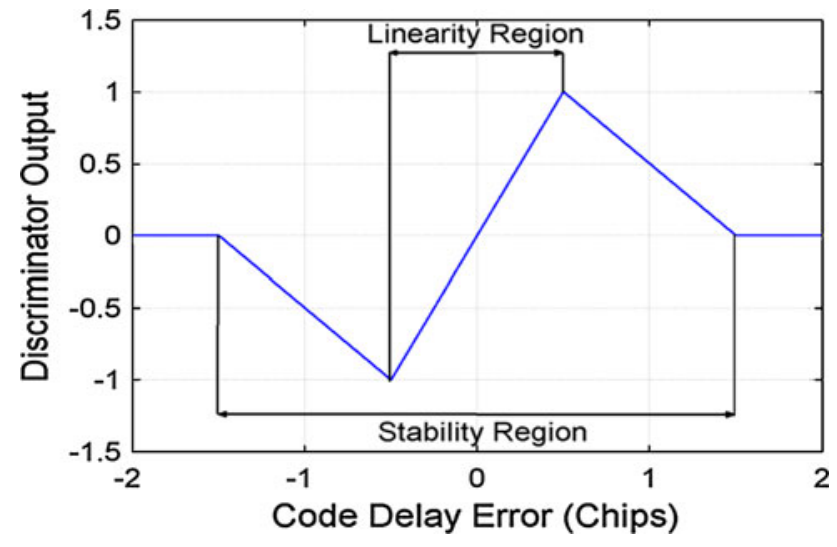

Fig. 7 Stability and linearity region for a typical discriminator output
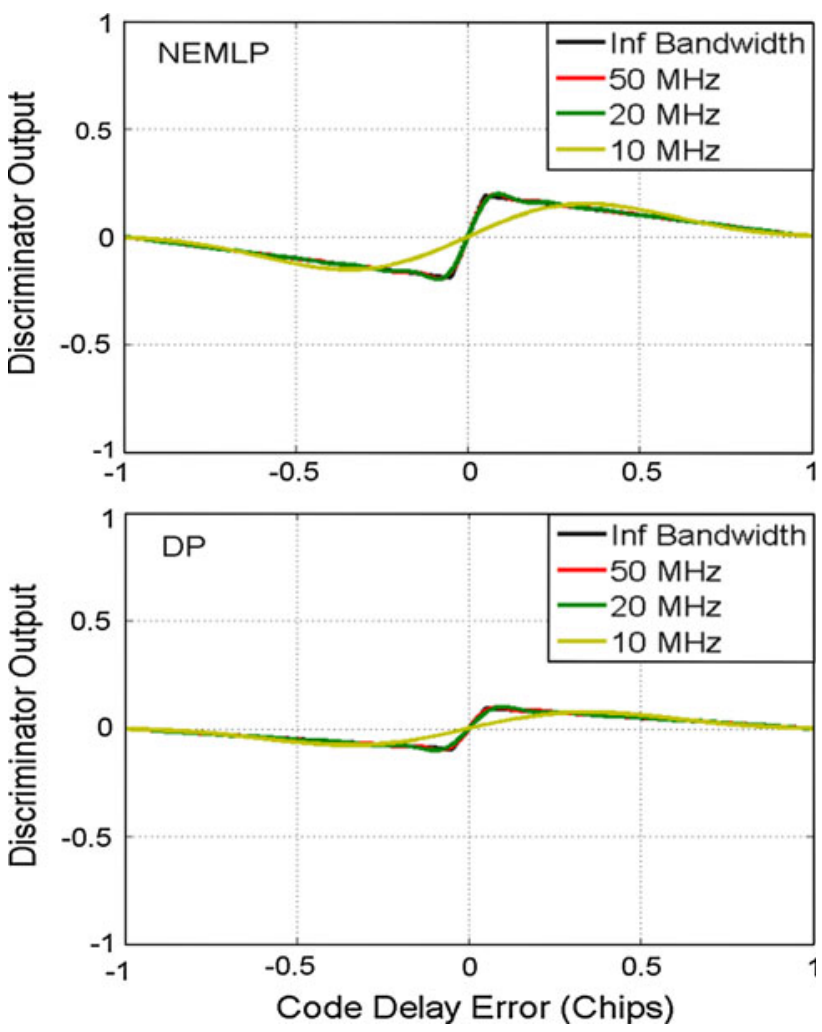

Fig. 8 NEMLP (top) and DP (bottom) discriminator output for E5a $\operatorname{BPSK}(10)$ for a correlator spacing of 0.1 chips

It is clear that the stability region for the BPSK(10) modulation ( \pm 1 chip) is much larger than for the AltBOC $(15,10)$ modulation $( \pm 0.167$ chip $)$, and this is due to its wider correlation peak and the non-existence of any secondary peak in its autocorrelation function (Fig. 4). This means that BPSK(10) is more robust and Alt$\operatorname{BOC}(15,10)$ is more sensitive to large code delay errors, which could potentially lead the latter to a false lock point. For both modulations, decreasing the front-end filter 

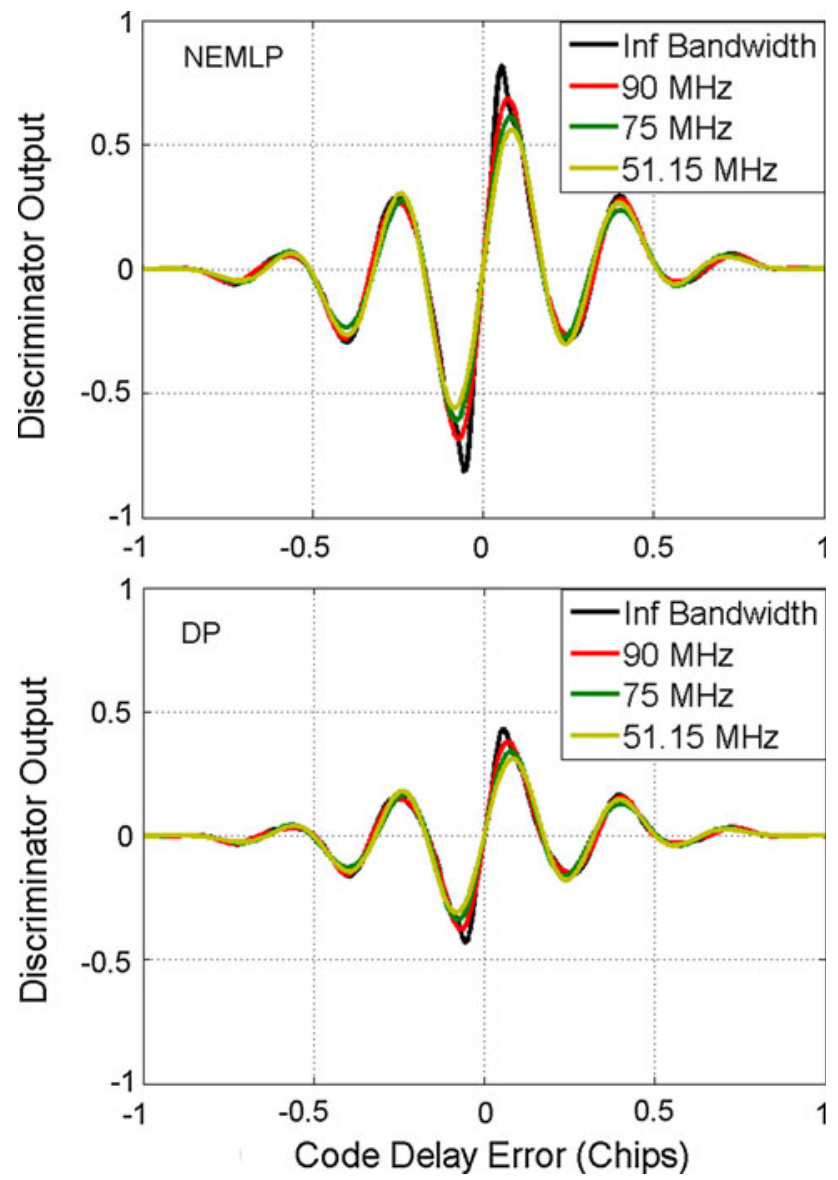

Fig. 9 NEMLP (top) and DP (bottom) discriminator output for E5 $\operatorname{AltBOC}(15,10)$ for a correlator spacing of 0.1 chips

bandwidth does not affect the stability region. However, the discriminator's gain known also as the discriminator's slope at the origin is affected. For $\operatorname{BPSK}(10)$, if the bandwidth is sufficiently large to cover the main lobe $(\geq 20 \mathrm{MHz}$ ), the discriminator slope will match the infinite bandwidth case. For AltBOC, the slope decreases with a decreasing bandwidth. It is also important to note that in all cases AltBOC $(15,10)$ has a better discriminator gain then $\operatorname{BPSK}(10)$.

Regarding the linearity, both signals have almost similar linear region situated around \pm 0.05 chips or $\pm d / 2$. In the case of BPSK(10), again assuming a sufficiently large enough bandwidth, the discriminator shape will match the infinite case. For the AltBOC, the front-end filter bandwidth round offs the correlation peak, and decreases its amplitude, and consequently the linearity slightly decreases as it can be seen from Fig. 9. It is important to mention that linearity is a very important criterion for tracking robustness. A narrow linear region means that the discriminator is more susceptible to input error larger than the linearity region, and in this case, the response will be biased. Usually, the linearity is dependent on the correlators spacing and the shape of the autocorrelation function. Figures 10 and 11 show the discriminator's outputs as a function of the correlator's spacings. In the case of BPSK(10) modulation, the linearity tracking region follows the theory that the linearity region should be equal to the chip spacing and it is contained within $\pm d / 2$ chips for the NEMLP discriminator. For the DP, with increasing the chip spacing, the discriminator starts to round off on the edge of the linearity region, which leads to a biased output and incorrect response. Hence, NEMLP discriminator is more robust and performs better with $\operatorname{BPSK}(10)$ modulation.

In the case of the $\operatorname{AltBOC}(15,10)$ modulation, the maximum linearity region obtained with the DP is within \pm 0.165 chips. This is due to the fact that the discriminator requires a correlator spacing value that is smaller than half of the one-sided width of the autocorrelation main peak (Julien 2005), corresponding to 1 chip for the $\operatorname{BPSK}(10)$ case and to 0.33 chips for the $\operatorname{AltBOC}(15,10)$. This is shown in the bottom graph of Fig. 11 where it is clear that for a correlator spacing higher than 0.33 (dashed lines), the discriminator output starts to be biased. In the case of NEMLP, the discriminator squares the autocorrelation function and consequently the two negative side peaks become positive, and the width of the main peak is decreased to 0.17 chips (Fig. 4). Thus, the maximum correlator spacing that can be used so that the NEMLP discriminator functions properly is 0.17 chips, and hence, the maximum linearity obtained is within \pm 0.085 chips. This is shown on the top graph of Fig. 11 where it is clear that for a correlator spacing higher than 0.17 chips (dashed lines), the discriminator output starts to be biased. Hence, a DP discriminator provides more robustness and better performance for AltBOC $(15,10)$.

In conclusion, BPSK(10) offers more freedom than AltBOC $(15,10)$ for choosing the correlator spacing, resulting in a wide linear tracking region that provides an improvement in tracking robustness and stability.

\section{Normalized DLL}

As shown before, the discriminators output does not directly estimate the true input code delay error. Consequently, to obtain an unbiased response, a normalization should be applied to the discriminator. Usually, the discriminator is normalized by its gain or its slope at the origin. However, this process exhibits a bias that will cause the discriminator to overestimate the error, when it starts to increase. Hence, two common normalization techniques 

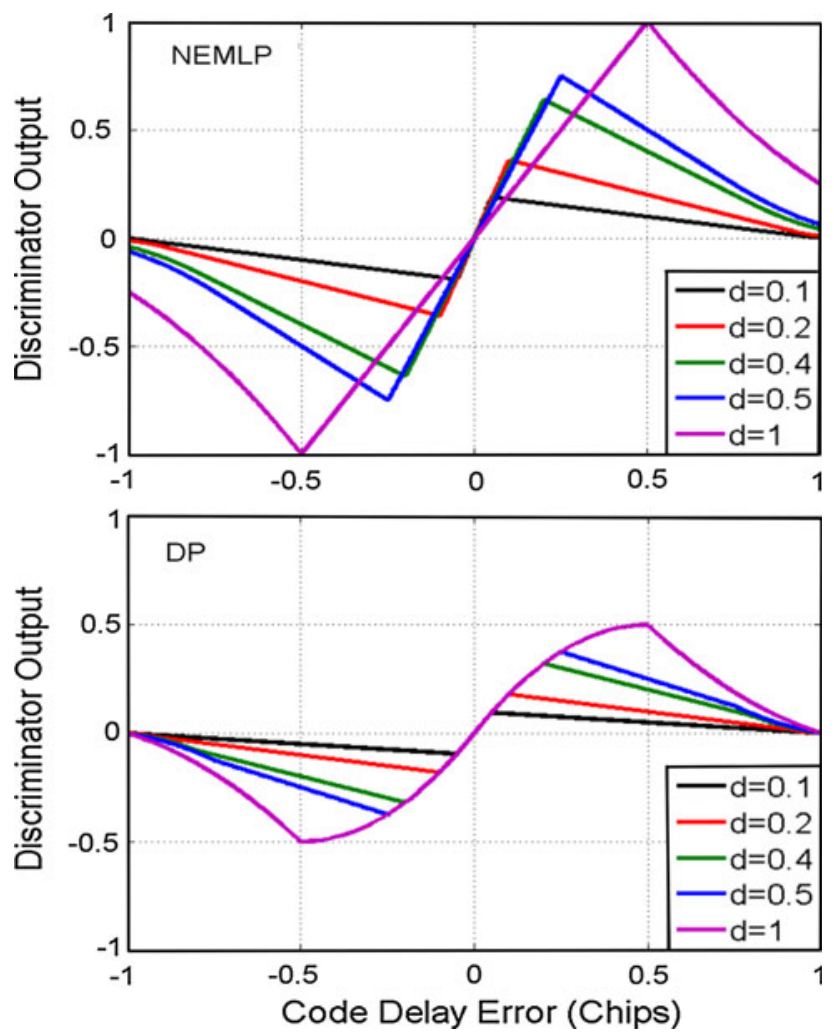

Fig. 10 NEMLP (top) and DP (bottom) discriminator output for E5a $\operatorname{BPSK}(10)$ as a function of correlator spacing for a $30-\mathrm{MHz}$ bandwidth

are often used for the NEMLP and DP discriminators as given by (Kaplan and Hegarty 2006):

$N_{\mathrm{NEMLP}}=\left(I_{\mathrm{E}}+I_{\mathrm{L}}\right)^{2}+\left(Q_{\mathrm{E}}+Q_{\mathrm{L}}\right)^{2}$
$N_{\mathrm{DP}}=\left(I_{\mathrm{E}}+I_{\mathrm{L}}\right) I_{\mathrm{P}}+\left(Q_{\mathrm{E}}+Q_{\mathrm{L}}\right) Q_{\mathrm{P}}$

To study the effect of these two normalizations, the output of the normalized discriminators should be derived. The autocorrelation function in (18) and the E5a autocorrelation can be modeled within the width of the main peak as the following:

$R(\tau)=1-\alpha(\tau)|\tau|$

where $\alpha(\tau)$ is the slope of the autocorrelation function. For $\operatorname{BPSK}(10), \alpha(\tau)=1$, and for an infinite bandwidth $\operatorname{AltBOC}(15,10)$, it is equal to:

$\alpha(\tau)=\cos \left(2 \pi f_{\mathrm{sc}} \tau\right)-2 \pi f_{\mathrm{sc}}(1-|\tau|) \sin \left(2 \pi f_{\mathrm{sc}} \tau\right)$

The outputs of the NEMLP and DP discriminators are then equal to:

$D_{\mathrm{NEMLP}}=\frac{A^{2}}{2} \alpha(-d / 2)(2-\alpha(-d / 2) d) \tau$
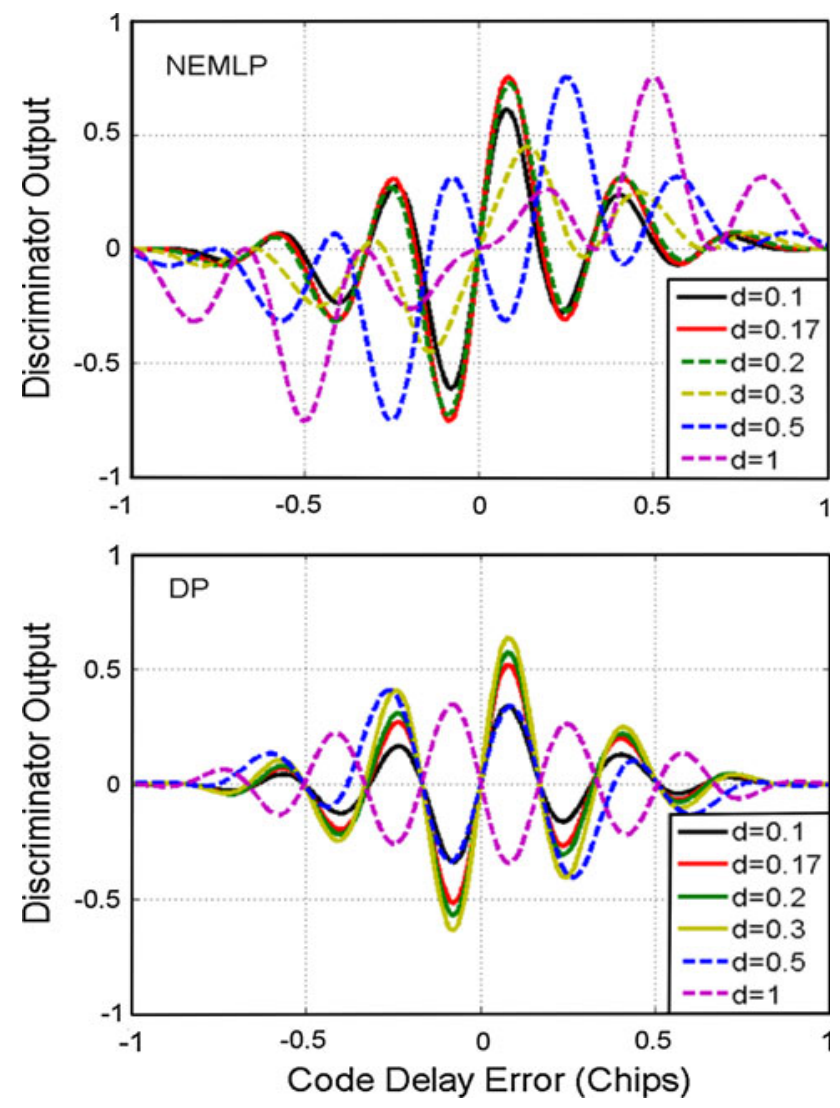

Fig. 11 NEMLP (top) and DP (bottom) discriminator output for E5 $\operatorname{AltBOC}(15,10)$ as a function of correlator spacing for a $75-\mathrm{MHz}$ bandwidth

$D_{\mathrm{DP}}=\frac{A^{2}}{2} \alpha(-d / 2)(2-\alpha(-d / 2)|\tau|) \tau$

where $\alpha(-d / 2)$ is the slope of the correlation peak, evaluated at $\tau=-d / 2$. Equations 27 and 28 can thus be written as:

$N_{\mathrm{NEMLP}}=\frac{A^{2}}{4}(2-\alpha(-d / 2) d)^{2}$

$N_{\mathrm{DP}}=\frac{A^{2}}{4}(2-\alpha(-d / 2) d)(1-\alpha(-d / 2)|\tau|)$

Finally, the output of the normalized discriminators is equal to:

$D_{\mathrm{NEMLP}}^{\text {Normalized }}=\frac{(2-\alpha(-d / 2) d) D_{\mathrm{NEMLP}}}{2 \alpha(-d / 2) N_{\mathrm{NEMLP}}} \approx \tau$

$D_{\mathrm{DP}}^{\text {Normalized }}=\frac{(2-\alpha(-d / 2) d) D_{\mathrm{DP}}}{2 \alpha(-d / 2) N_{\mathrm{DP}}} \approx \tau$

Figures 12 and 13 show the output of the normalized NEMLP and DP discriminators for $\operatorname{BPSK}(10)$ and AltBOC $(15,10)$ modulations. In all cases, the stability region is not affected by the normalization. 

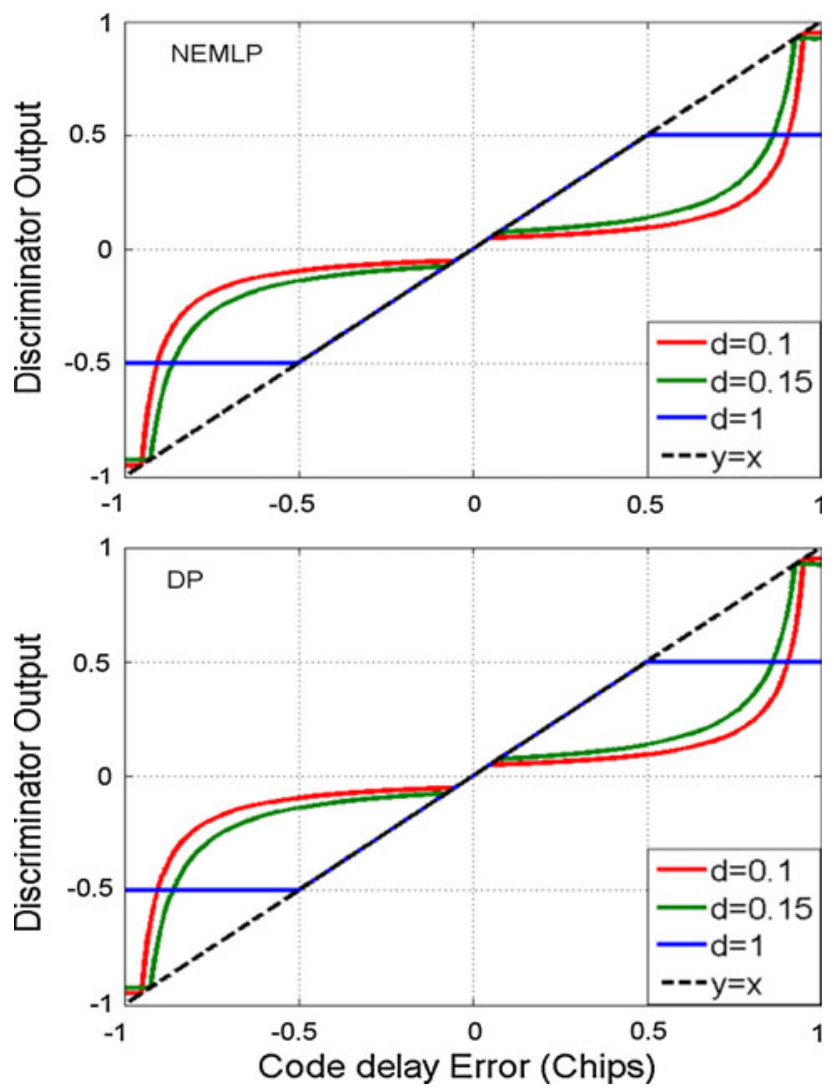

Fig. 12 Normalized NEMLP (top) and DP (bottom) discriminator output for E5a BPSK(10) as a function of correlator spacing for a 30-MHz bandwidth

For BPSK(10), it can be seen that both discriminators have very similar output and a linear tracking region within $\pm d / 2$ chips. Outside this region, both discriminators will underestimate the code tracking error. One advantage of normalization for the DP with the $\operatorname{BPSK}(10)$ is that the discriminator has a linear output even with increasing the code delay error in contrary to the case where no normalization is used. In the case of $\operatorname{AltBOC}(15,10)$, both discriminators have also very similar shapes, and it can be seen that the linearity region is wider than $\pm d / 2$ chips. In fact, for 0.1 and 0.15 chip spacing, the linearity region should be within \pm 0.05 and \pm 0.075 chips, but a closer look on Fig. 13 shows that for both discriminators the linearity region has been extended to \pm 0.1 and \pm 0.12 chips, respectively. This shows that with normalization the AltBOC $(15,10)$ modulation shows a more robust tracking comparing with BPSK(10) for the same chip spacing where the discriminator will act without bias even for code errors higher than $\pm d / 2$ chips.

It is important to mention that for both modulations and discriminators at the edge of the stability regions, the tracking starts to be unstable due to the existence of vertical asymptotes that can lead to large errors or false lock points if a sudden tracking jump occurs. Usually,
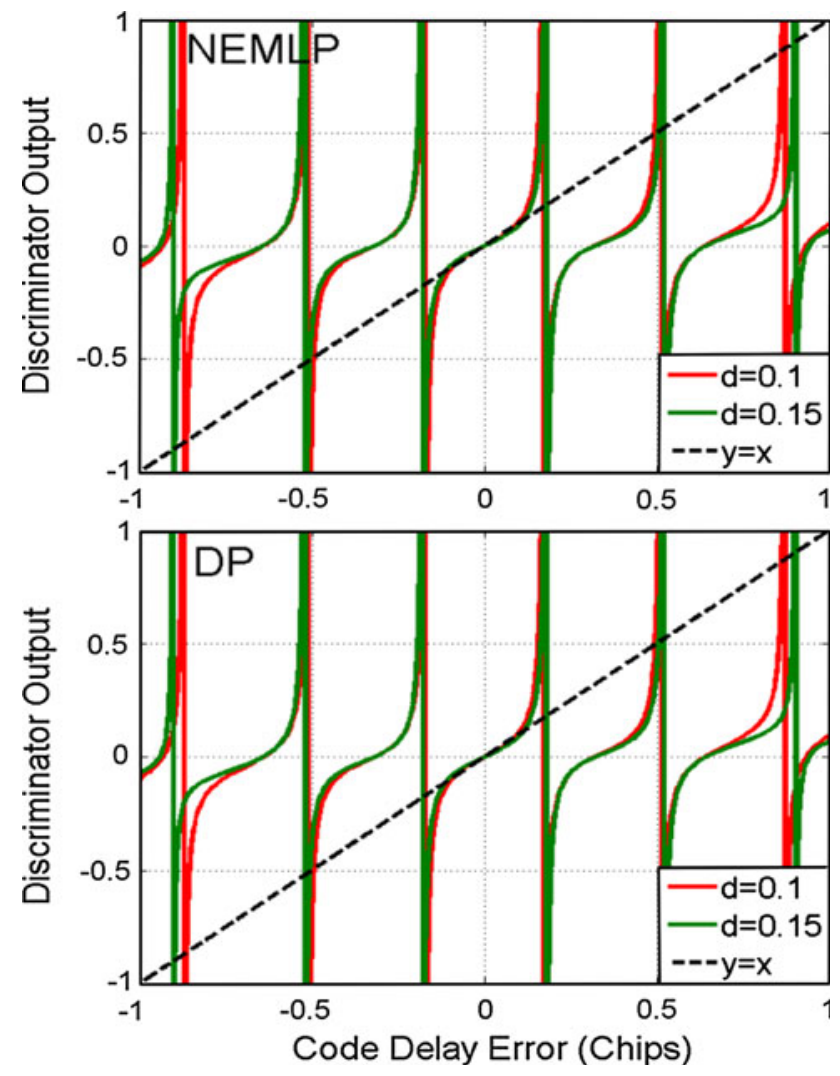

Fig. 13 Normalized NEMLP (top) and DP (bottom) discriminator output for $\mathrm{E} 5 \operatorname{AltBOC}(15,10)$ as a function of correlator spacing for a 75-MHz bandwidth

Table 3 Maximum stability and linearity regions in chips obtained with normalized and unnormalized NEMLP and DP discriminators for E5 and E5a pilot channels

\begin{tabular}{llllll}
\hline & \multicolumn{2}{l}{ AltBOC $(15,10)$} & & \multicolumn{2}{l}{ BPSK(10) } \\
\cline { 2 - 3 } \cline { 5 - 6 } & Stability & Linearity & & Stability & Linearity \\
\hline NEMLP/ & \pm 0.172 & \pm 0.085 & & \pm 1 & \pm 0.5 \\
norm & \pm 0.172 & \pm 0.133 & & \pm 1 & \pm 0.5 \\
DP/ & \pm 0.172 & \pm 0.165 & & \pm 1 & \pm 0.2 \\
norm & \pm 0.172 & \pm 0.165 & & \pm 1 & \pm 0.5 \\
\hline
\end{tabular}

carrier aiding can control these jumps and limit their threats. Finally, Table 3 shows the maximum stability and linearity regions that can be obtained with normalized and unnormalized discriminators for $\operatorname{AltBOC}(15,10)$ and $\operatorname{BPSK}(10)$.

In conclusion, a NEMLP normalized discriminator has a better performance with BPSK(10), and DP normalized is more suitable for $\operatorname{AltBOC}(15,10)$. In terms of tracking robustness, BPSK(10) offers a large stability region and more choices in correlator spacing than $\operatorname{AltBOC}(15,10)$, but the latter has better linearity for the same chip spacing. 


\section{Code tracking errors}

In a tracking loop, three main errors exist as follows: thermal noise, multipath, and signal dynamics. The dynamic stress is due to satellite-receiver motion and mainly controlled by the PLL and carrier aiding to the DLL. In this case, the resulting induced error is negligible comparing with the other two error sources and consequently, it will not be addressed in this section.

\section{Code noise error}

In the absence of multipath or other distortion of the received signal and no interference, the dominant sources of range error in a GNSS receiver code tracking loop (DLL) are thermal noise range error. The noise corresponds to the ambient noise present at the receiver antenna. The general expressions for thermal noise code tracking jitter for a NEMLP and DP discriminators are given by Ries et al. (2002) and Betz (2000):

$$
\begin{gathered}
\sigma_{\mathrm{NEMLP}}^{2}=\frac{B_{n} \int_{-B / 2}^{B / 2} G(f) \sin ^{2}(\pi f d) d f}{\frac{C}{N_{0}}\left(2 \pi \int_{-B / 2}^{B / 2} f G(f) \sin (\pi f d) d f\right)^{2}} \beta \\
\sigma_{\mathrm{DP}}^{2}=\frac{B_{n} \int_{-B / 2}^{B / 2} G(f) \sin ^{2}(\pi f d) d f}{\frac{C}{N_{0}}\left(2 \pi \int_{-B / 2}^{B / 2} f G(f) \sin (\pi f d) d f\right)^{2}} \psi
\end{gathered}
$$

where $\beta$ and $\psi$ are equal to:

$$
\begin{aligned}
& \beta=\left(1+\frac{\int_{-B / 2}^{B / 2} G(f) \cos ^{2}(\pi f d) d f}{T_{\operatorname{int}} \frac{C}{N_{0}}\left(\int_{-B / 2}^{B / 2} G(f) \cos (\pi f d) d f\right)^{2}}\right) \\
& \psi=\left(1+\frac{1}{T_{\operatorname{int}} \frac{C}{N_{0}}\left(\int_{-B / 2}^{B / 2} G(f)\right)}\right)
\end{aligned}
$$

$G(f)$ is the power spectral density of the received signal, $C / N_{0}$ is the carrier-to-noise ratio, $B_{n}$ is the code loop noise bandwidth (equal to $1 \mathrm{~Hz}$ in the following), and $\mathrm{B}$ is the front-end bandwidth. Replacing $G(f)$ by (4), the code noise errors for the E5 pilot channel assuming a ' $d$ ' chip spacing can be derived as:

Table 4 AltBOC $(15,10)$ autocorrelation slope (i.e. $\alpha$ ) evaluated at $d / 2$ for different front-end bandwidths and chip spacings

\begin{tabular}{llll}
\hline$B$ & $90 \mathrm{MHz}$ & $75 \mathrm{MHz}$ & $51.15 \mathrm{MHz}$ \\
\hline$d=0.1$ & 4.675 & 3.965 & 3.533 \\
$d=0.15$ & 6.276 & 5.528 & 4.996 \\
$d=0.2$ & 7.137 & 6.631 & 6.122 \\
$d=0.3$ & 7.382 & 7.251 & 7.114 \\
\hline
\end{tabular}

$\sigma_{\mathrm{NEMLP}}^{2}=\frac{B_{n}\left(1-\tilde{R}_{Q}(d)\right)}{2 \alpha_{(-d / 2)}^{2} \frac{C}{N_{0}}}\left(1+\frac{2}{\left(2-\alpha_{(-d / 2)} d\right) \frac{C}{N_{0}} T_{\mathrm{int}}}\right)$

$\sigma_{\mathrm{DP}}^{2}=\frac{B_{n}\left(1-\tilde{R}_{Q}(d)\right)}{2 \alpha_{(-d / 2)}^{2} \frac{C}{N_{0}}}\left(1+\frac{1}{\frac{C}{N_{0}} T_{\mathrm{int}}}\right)$

The code error for E5a pilot BPSK(10) signal has a similar expression with a constant autocorrelation slope $\alpha=1$. For $\operatorname{AltBOC}(15,10)$, the autocorrelation slope is a function of the chip spacing $\mathrm{d}$ and the front-end bandwidth. Table 4 shows the values of $\alpha$ evaluated at $d / 2$. It can be noted that when decreasing the bandwidth the slope decreases, and the amount of decreasing is lower when increasing the chip spacing.

Equations 41 and 42 show that the DP discriminator has a better performance in terms of code noise error than the NEMLP discriminator, due to the squaring of the early and late correlators. They also indicate that this loss is higher for $\operatorname{AltBOC}(15,10)$ as the slope of its correlation function is higher than BPSK(10). Figure 14 shows the impact of the front-end filter bandwidth on the code noise error for the AltBOC $(15,10)$ modulation. It can be seen that when decreasing the bandwidth, the code noise increases. The DP discriminator is more sensitive to a smaller bandwidth as when lowering from 90 to 75 and 51.15, an average loss of $0.45 \mathrm{~dB}$ is obtained at each step. With the NEMLP, an average loss of $0.31 \mathrm{~dB}$ is obtained at each step. As for the $\operatorname{BPSK}(10)$, and as discussed elsewhere, as long as the frontend filter is large enough $(\geq 20 \mathrm{MHz})$ to cover the main lobe of the PSD, the impact on the discriminator function is very low, and hence, the impact on the code noise error is negligible.

The impact of early/late chip spacing is shown in Fig. 15 for both modulations and for a 1-ms integration time with a $75-\mathrm{MHz}$ bandwidth for $\operatorname{AltBOC}(15,10)$ and $20 \mathrm{MHz}$ for BPSK(10). For the AltBOC modulation, the DP discriminator is less sensitive to correlator spacing than for the NEMLP, where a loss of $0.31 \mathrm{~dB}$ in code noise error is obtained for increasing the chip spacing from 0.1 to 0.15 and $0.85 \mathrm{~dB}$ from 0.15 to 0.2 . Consequently, with the $\mathrm{DP}$, a loss of 0.04 and $0.087 \mathrm{~dB}$ is obtained.

It is important to also note that even with 0.2 chip spacing, the DP discriminator performs better than the NEMLP with 0.1 chip spacing. For the BPSK(10) modulation, the DP and NEMLP have very similar performance, and they are more sensitive to chip spacing. The code noise loss is $1.53 \mathrm{~dB}$ going from 0.1 to 0.2 chip spacing and $0.91 \mathrm{~dB}$ from 0.2 to 0.3 chip spacing. Finally, Fig. 16 shows the code noise error for both modulations for 0.1 chip spacing. It is clear that $\operatorname{AltBOC}(15,10)$ has a better resistance to noise and $\operatorname{BPSK}(10)$ has an average loss of 


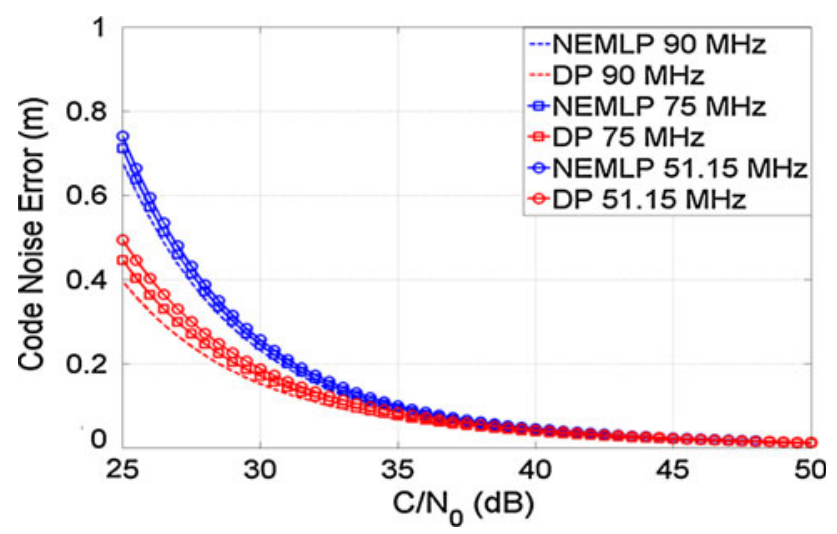

Fig. 14 Impact of the front-end filter bandwidth on the Alt$\operatorname{BOC}(15,10)$ code noise error for a 0.2 chip spacing and 1-ms integration time
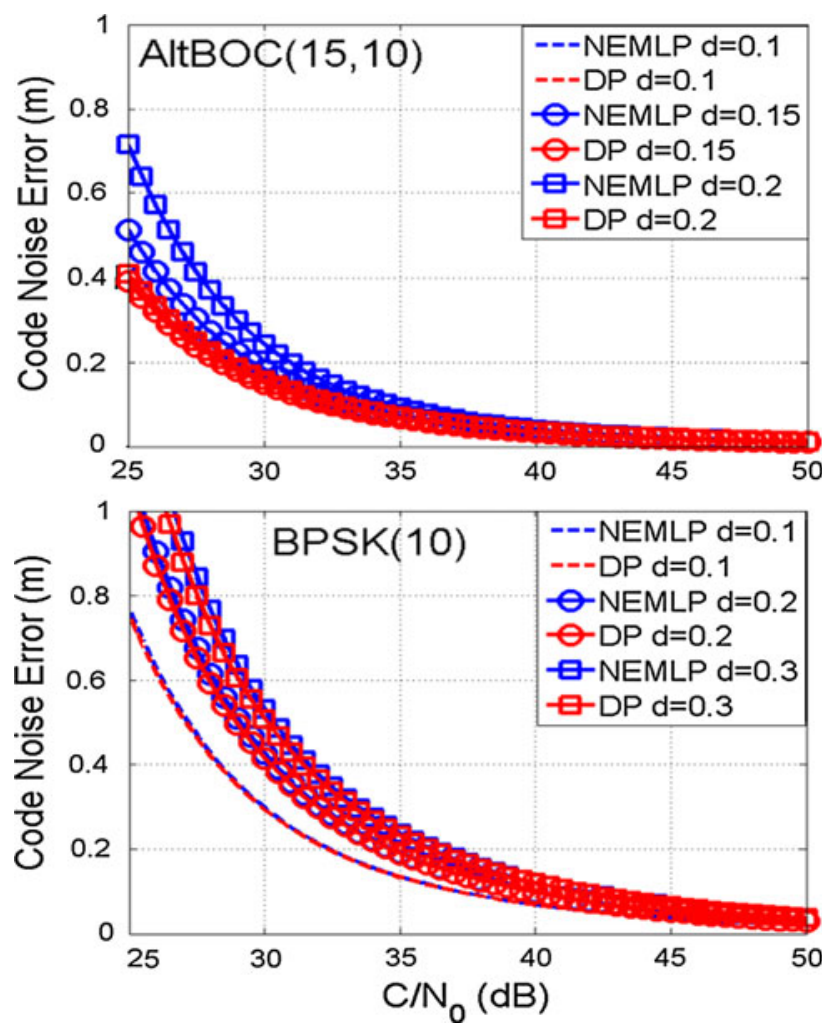

Fig. 15 Impact of the early-late chip spacing on the code noise error for $\operatorname{AltBOC}(15,10)$ and $\operatorname{BPSK}(10)$ for a $75-$ and $30-\mathrm{MHz}$ front-end filter bandwidth, respectively

$2 \mathrm{~dB}$ in code noise error. This loss will increase when increasing the chip spacing.

In conclusion, it was shown that the DP discriminator has a better performance and is less sensitive to chip spacing for AltBOC $(15,10)$. For BPSK(10), both discriminators are sensitive to chip spacing and have almost the same code noise error. Also, it was shown that $\operatorname{AltBOC}(15,10)$

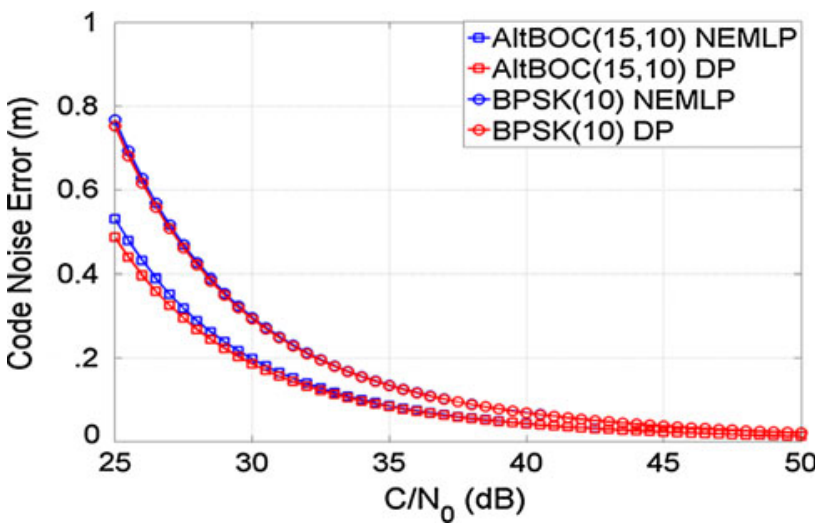

Fig. 16 Code noise error for $\operatorname{AltBOC}(15,10)$ and $\operatorname{BPSK}(10)$ for a 0.1 chip spacing and 1-ms integration time

outperforms BPSK(10) and has an average of 2-dB gains in tracking error standard deviation.

Code multipath error

In a GNSS receiver, multipath is caused by reflections of satellite signals from nearby objects, buildings, or ground. These reflected signals arrive with an additional delay as compared with the line of sight (LOS) path. In a GPS or Galileo receiver, the only desired signal to measure the pseudorange is the direct one as all the other multipath signals may cause important ranging and carrier phase errors (Tawk et al. 2010). In fact, with the presence of reflected signals arriving at the receiver after multiple reflections, diffraction, and scattering, the baseband signal, the discriminator output, and the correlation function may all be distorted. As the pseudoranges are obtained from these quantities, the distortions may lead to a false or biased lock and consequently to a range and phase error. The impact of multipath on code tracking accuracy is often represented as an error envelope representing the maximum error resulting from one single multipath with a certain phase, delay, and amplitude. It is called multipath error envelope (MEE). It is important to note that, when computing the multipath-induced code tracking error envelope, it is equivalent to finding the point of the zerocrossing position of the total path discriminator output function, since this means that it represents the stability point where the loop will lock and gives the code phase multipath error (Jovanovic et al. 2010). The NEMLP and DP discriminators have the same performance in terms of multipath mitigation as the difference in zero crossing between the two discriminators is the same. Figure 17 shows the code tracking error envelope for $\operatorname{AltBOC}(15,10)$ and $\operatorname{BPSK}(10)$ for a received signal with one reflection having a signal-to-multipath ratio (SMR) of $3 \mathrm{~dB}$. 


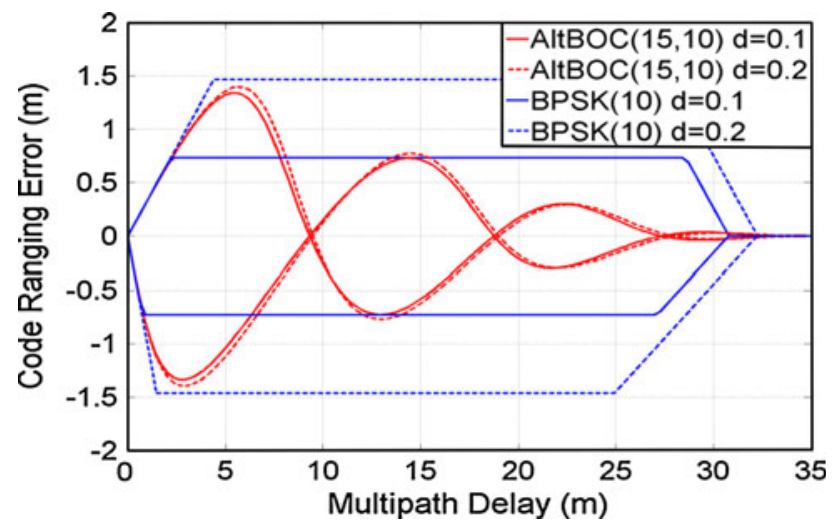

Fig. 17 Multipath error envelopes for $\operatorname{AltBOC}(15,10)$ and $\operatorname{BPSK}(10)$ signals for different correlator spacing

In general, a narrow correlator spacing results in a better multipath mitigation. For the E5 AltBOC modulation, it is less sensitive to correlator spacing, in contrast to $\operatorname{BPSK}(10)$, where an increase in correlator spacing from 0.1 to 0.2 chips increases the code multipath error around $3 \mathrm{~dB}$. Another metric for multipath assessment is the computation of the running average (i.e. average error for a specific multipath delay) of multipath code error envelopes where a good multipath performance is characterized by a small maximum average and a rapid decrease toward zero for a short multipath delay. Figure 18 shows the code error running averaged for $\operatorname{AltBOC}(15,10)$ and $\operatorname{BPSK}(10)$.

For 0.1 chip spacing, the BPSK(10) provides better mitigation than $\operatorname{AltBOC}(15,10)$ for short delay multipath signals $(\leq 11 \mathrm{~m})$, but the latter performs better for long delays. With the increase in the correlator spacing, Alt$\operatorname{BOC}(15,10)$ has a better mitigation for all the delays. It is important to note that $\operatorname{AltBOC}(15,10)$ has a faster decrease toward zero where the maximum error occurs around 7-9 $\mathrm{m}$ delays and it starts to decrease rapidly, while for BPSK(10) the maximum error occurs around 27-29 m delays and starts to decrease slowly. To conclude, from Figs. 17 and 18, it can be seen that the multipath-induced error is a dominant error in tracking loops and higher than code noise error. E5 pilot signal has a better mitigation than E5a pilot, but for narrow correlators (i.e. $\leq 0.15$ chips), the improvement is very negligible and for short delays the E5a has a better performance.

\section{Tracking sensitivity}

The tracking loop sensitivity is defined as the minimum carrier-to-noise ratio $\left(C / N_{0}\right)$ required by the receiver to be

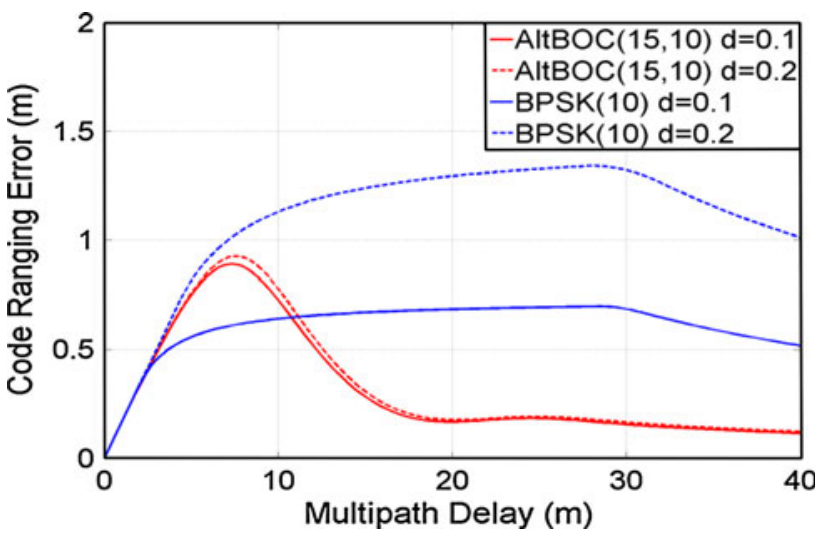

Fig. 18 Multipath code error running average for $\operatorname{AltBOC}(15,10)$ and BPSK(10) signals for different correlator spacing

able to continue tracking. One of the main parameters that increase the tracking sensitivity is the coherent integration time, and with the availability of pilot channels on both E5 and E5a signals, it is theoretically possible to integrate for very long periods of time, thus providing a significant increase in the post-correlation SNR and consequently an increase in the sensitivity. The rule-of-thumb tracking threshold for the DLL is that the 3-sigma value of the jitter due to all sources of loop error must not exceed half of the linear pull-in range of the discriminator (Kaplan and Hegarty 2006). Therefore, the tracking threshold is defined as:

$3 \sigma_{\mathrm{DLL}} \leq \frac{L}{2}$

where $L$ is the linearity region, and $\sigma_{\mathrm{DLL}}$ is equal to the error induced by the thermal noise and dynamic stress. However, as with carrier aiding the dynamic stress error in the DLL tracking loop is negligible, then it will not be included herein. It is also important to note that the multipath-induced error is not included as it is considered as a bias that shifts the discriminator output stable point. However, it does not imply a tracking error that would push the tracking loop away from its stability point or would affect the linearity region (Julien 2005). Consequently, for a single multipath, it cannot be considered as directly affecting the code tracking sensitivity. Using (41) and (42), the resulting tracking threshold can be modeled as:

$\frac{C}{N_{0}} \geq \frac{18 A}{L^{2}}\left(1+\sqrt{1+\frac{L^{2} B}{9 A}}\right)$

where $A$ and $B$ are equal to:

$A=\frac{B_{n}\left(1-\tilde{R}_{Q}(d)\right)}{2 \alpha_{(-d / 2)}^{2}}$ 


$$
\begin{aligned}
& B_{\mathrm{NEMLP}}=\frac{2}{\left(2-\alpha_{(-d / 2)} d\right) T_{\mathrm{int}}} \\
& B_{\mathrm{DP}}=\frac{1}{T_{\mathrm{int}}}
\end{aligned}
$$

The resulting tracking thresholds for different receiver bandwidths and for a correlator spacing of 0.1 chips are shown in Fig. 19 for Galileo E5 and E5a pilot channels. It can be seen that a higher sensitivity can be achieved with the Galileo E5 pilot $\operatorname{AltBOC}(15,10)$ signal. For the minimum required bandwidth to receive both signals (i.e. 51.15 and $20 \mathrm{MHz})$, the $\operatorname{AltBOC}(15,10)$ with the DP discriminator has a tracking threshold between 2.62 and $4.43 \mathrm{~dB}$ higher than the BPSK(10). Also as expected, with the DP discriminator, the tracking thresholds are $0.2 \mathrm{~dB}$ in average higher than for the NEMLP in the case of $\operatorname{AltBOC}(15,10)$, and for $\operatorname{BPSK}(10)$, both discriminators have almost the same thresholds. The impact of the correlator spacing on the tracking thresholds is shown in Fig. 20 for the DP discriminator and a minimum required bandwidth to receive both signals. It is clear that with increasing the chip spacing, the linearity region increases and consequently the threshold decreases. For AltBOC $(15,10)$ and as shown in 'Normalized DLL' section, the linearity region is wider than for the BPSK(10) for the same correlator spacing and thus the decrease in the tracking thresholds is higher. An improvement of around $6.84 \mathrm{~dB}$ is obtained by increasing the correlator spacing from 0.1 to 0.2 chips and $3.32 \mathrm{~dB}$ by increasing it from 0.2 to 0.3 chips. For $\operatorname{BPSK}(10)$, the improvement is 4.21 and $1.67 \mathrm{~dB}$, respectively.

\section{Summary and conclusions}

Throughout this work, the characteristics of tracking loops for the Galileo E5 signal were presented. It has been shown that the E5 signal can be tracked in two modes as follows: as an $\operatorname{AltBOC}(15,10)$ modulation or as a $\operatorname{BPSK}(10)$ like modulation. The impact of many parameters that affect the DLL loop, such as the front-end filter bandwidth, the correlator spacing, discriminator type, and normalization technique, was discussed. The performance for the NEMLP and DP discriminators with the $\operatorname{AltBOC}(15,10)$ and BPSK(10) modulation was analyzed, and it was shown that a DP discriminator is more suitable for $\operatorname{AltBOC}(15,10)$ and a NEMLP discriminator has a slightly better performance with BPSK(10). The advantages and drawbacks of tracking both modulations as compared to each other were also assessed and can be summarized as.

Tracking E5 as an $\operatorname{AltBOC}(15,10)$ presents several advantages over BPSK(10). For the same correlator spacing that is smaller than half of the one-sided width of the autocorrelation main peak, the $\operatorname{AltBOC}(15,10)$ has a wider
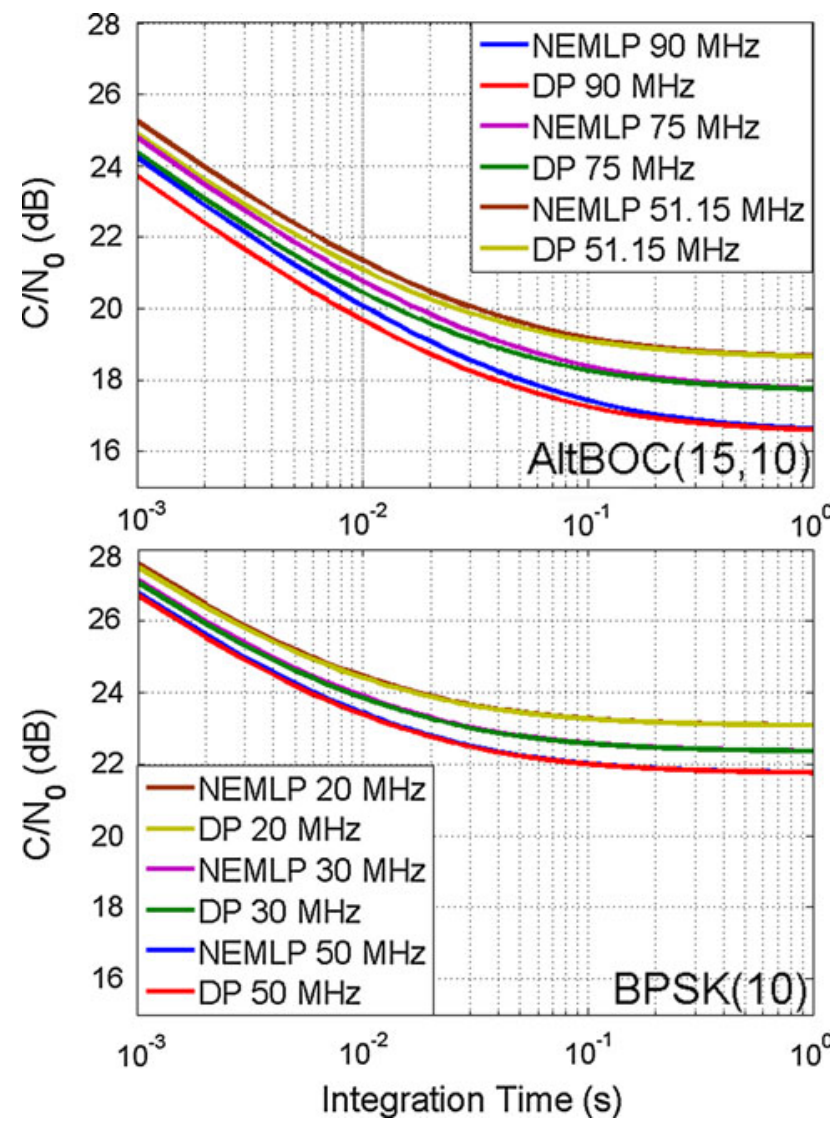

Fig. 19 Impact of the receiver bandwidth on the tracking thresholds for $\operatorname{AltBOC}(15,10)$ and $\operatorname{BPSK}(10)$ for a 0.1 chip correlator spacing

linearity region than BPSK(10) using either the NEMLP or DP discriminator. Also, an average improvement of around $2 \mathrm{~dB}$ in code noise error standard deviation is obtained assuming the same loop settings. The $\operatorname{AltBOC}(15,10)$ showed a better multipath mitigation for long delays and for short delays with a correlator spacing higher than 0.15 chips. Moreover, an improvement in tracking threshold from 3 to $6 \mathrm{~dB}$ is obtained, which make the $\operatorname{AltBOC}(15,10)$ modulation a very good candidate for high-sensitive receivers.

Although having these advantages, the $\operatorname{AltBOC}(15,10)$ contains several drawbacks as compared with BPSK(10). The wide spectrum of the $\operatorname{AltBOC}(15,10)$ modulation requires a large front-end filter bandwidth. Consequently, a high sampling frequency is a prerequisite, which will lead to the need of a more powerful processor to process the signal that will result in more power consumption. Correspondingly, the required correlator spacing should be smaller than half of the one-sided width of the autocorrelation main peak, meaning that the maximum spacing that could be used should be $\leq 0.33$ chips. Furthermore, the Galileo E5a (E5b) BPSK(10) has a stability domain $( \pm 1$ chip) larger than the $\operatorname{AltBOC}(15,10)( \pm 0.172$ chip $)$ and a 


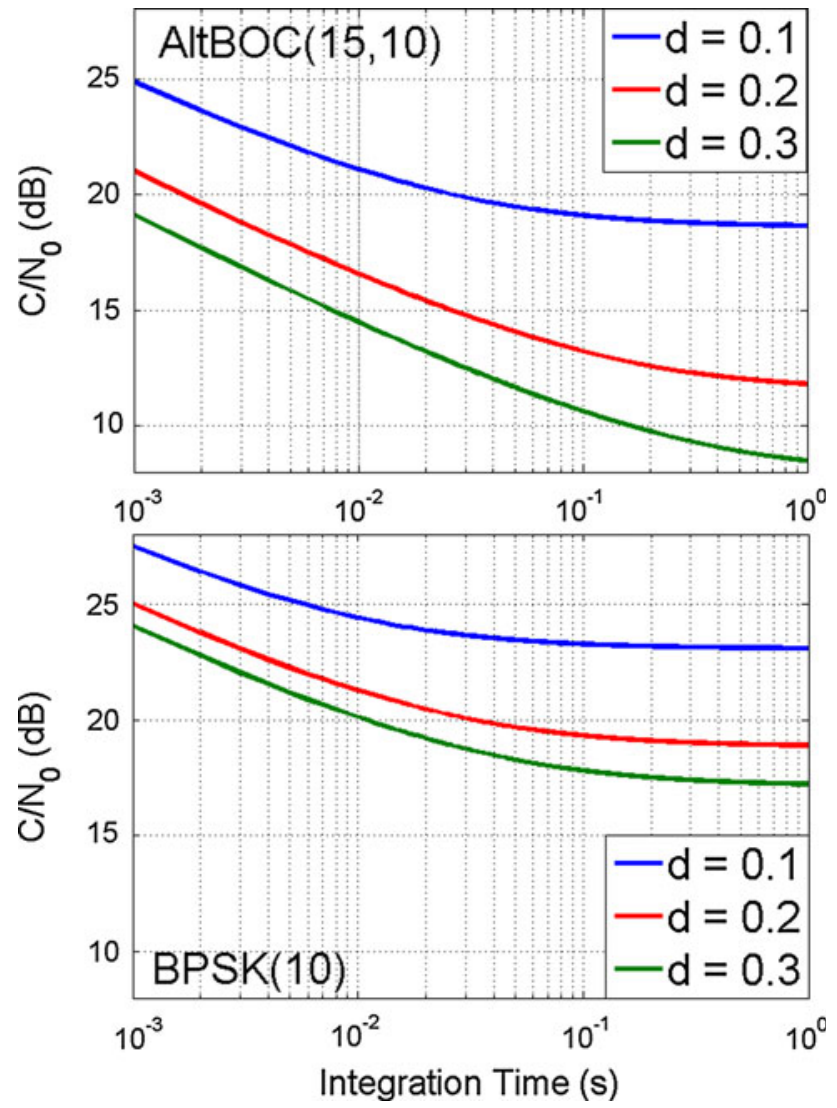

Fig. 20 Impact of the correlator spacing on the tracking thresholds for AltBOC $(15,10)(51.15 \mathrm{MHz})$ and $\operatorname{BPSK}(10)(20 \mathrm{MHz})$ with the DP discriminator

wider choice for correlator spacing's that could increase the linearity region up to \pm 0.5 chip. Additionally, the BPSK(10) showed more sensitivity to correlator spacing than the $\operatorname{AltBOC}(15,10)$, which could give the receiver designer more freedom in selecting the parameters for his design.

Finally, both modulations showed great potentials for robustness and accurate tracking loops. The existence of the pilot channel allows long integration time and improves the tracking gain. The high code PRN rate $(10.23 \mathrm{MHz})$ provides a good resistance to long multipath delays ( $\geq 35 \mathrm{~m}$ ) and better code noise error. In conclusion, depending on the receiver application, the designer could choose to track the E5 signal as a full band $\operatorname{AltBOC}(15,10)$ or a limited band BPSK(10) modulation. AltBOC $(15,10)$ is more suitable for high sensitive or robust application. BPSK(10) is a good candidate for low-cost receivers with low computational load and application requiring high stability.

Acknowledgments The authors are grateful for the financial support from the Swiss National Science Foundation (http://www.snf.ch/) who supports this work under grant 200021120187/1.

\section{References}

Avila-Rodriguez JA (2008) On generalized signal waveforms for satellite navigation. PhD Thesis, University FAF Munich, Faculty of Aerospace Engineering, Munich, Germany

Betz J (2000) Design and performance of code tracking for the GPS $M$ code signal. In: Proceedings of the 13th international technical meeting of the satellite division of the institute of navigation (ION GPS 2000), Salt Lake City, UT, pp 2140-2150, September 2000

Betz J, Kolodziejski K (2000) Extended theory of early-late code tracking for a bandlimited GPS receiver. Navig J 47(3):211-226

Braasch M, Dierendonck J (1999) GPS receiver architectures and measurements. Proc IEEE 87(1):48-64

European Space Agency, European Commission (2010) Galileo open service signal in space interface control document GAL OS SIS ICD issue 1

Gerein N (2005) Hardware architecture for processing Galileo alternate binary offset carrier (Altboc) signals. US Patent App. 10/681, 689

Jovanovic A, Tawk Y, Botteron C, Farine PA (2010) Multipath mitigation techniques for CBOC, TMBOC and AltBOC signals using advanced correlator architectures. In: Proceedings of IEEE/ ION position location and navigation symposium PLANS 2010, Indian Wells/Palm Springs, California, USA, pp 1127-1136

Julien O (2005) Design of Galileo L1F receiver tracking loops. PhD Thesis, published as UCGE report no. 20227, Department of Geomatics Engineering, The University of Calgary

Kaplan E, Hegarty C (2006) Understanding GPS principles and applications. Artech House, ISBN 1-58053-894-0

Lestarquit L, Artaud G, Issler J.L (2008) AltBOC for dummies or everything you always wanted to know about AltBOC. In: Proceedings of the 21st international technical meeting of the satellite division of the institute of navigation (ION GNSS 2008), Savannah, GA, pp 961-970, September 2008

Margaria D, Dovis F, Mulassano P (2007) An innovative data demodulation technique for Galileo AltBOC receivers. J Global Position Systems 6(1):89-96

Ries L, Lestarquit L, Armengou-Miret E, Legrand F, Vigneau W, Bourga C, Erhard P, Issler JL (2002) A software simulation tool for GNSS2 BOC signals analysis. In: Proceedings of the 15th international technical meeting of the satellite division of the institute of navigation (ION GPS 2002), Portland, OR, pp 2225-2239, September 2002

Shivaramaiah NC (2009) Code phase multipath mitigation by exploiting the frequency diversity in Galileo E5 AltBOC. In: Proceedings of the 22nd international technical meeting of the satellite division of the institute of navigation (ION GNSS 2009), Savannah, GA, pp 3219-3233, September 2009

Shivaramaiah NC, Dempster AG (2009) The Galileo E5 AltBOC: understanding the signal. In: Proceedings of the international global navigation satellite systems society IGNSS symposium 2009, Queensland, Australia

Shivaramaiah NC, Dempster AG, Rizos C (2009) Hybrid tracking loop architectures for the Galileo E5 signal. In: Proceedings of the European navigation conference on global navigation satellite systems ENC GNSS 2009, Napoli, Italy

Sleewaegen JM, De Wilde W, Hollreiser M (2004) Galileo AltBOC receiver. In: Proceedings of the European navigation conference on global navigation satellite systems ENC GNSS 2004, Rotterdam, Netherlands

Spirent (2008) GSS8000 multi-GNSS constellation simulator. www.spirent.com/Solutions-Directory/GSS8000.aspx

Tawk Y, Jovanovic A, Botteron C, Farine PA (2010) Performance comparison of different correlation techniques for the AltBOC modulation in multipath environments. In: Proceedings of IEEE 
international conference on communications ICC 2010, Cape Town, South Africa. doi: 10.1109/ICC.2010.5502625

\section{Author Biographies}
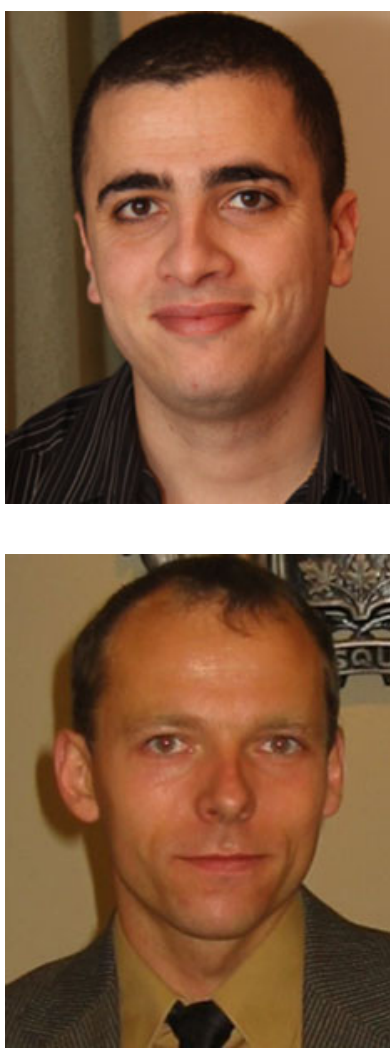

Youssef Tawk up to a PhD in the area of global navigation satellite system at EPFL. Graduated in 2007 from the University of Gavle in Sweden, he holds a Master's of Science degree in RF and Wireless Communication. In 2005, he received his Diploma in Electrical Engineering from the Lebanese University Faculty of Engineering. Currently, he is member of the GNSS group in the electronics and signal processing laboratory at EPFL.

Cyril Botteron leads the GNSS and UWB groups in the electronics and signal processing laboratory at EPFL. He received his $\mathrm{PhD}$ degree from the University of Calgary, Canada, in 2003. His current research interests comprise the development of low-power radio frequency (RF)-integrated circuits and advanced signal processing techniques for ultra-low power communications and global and local positioning applications.

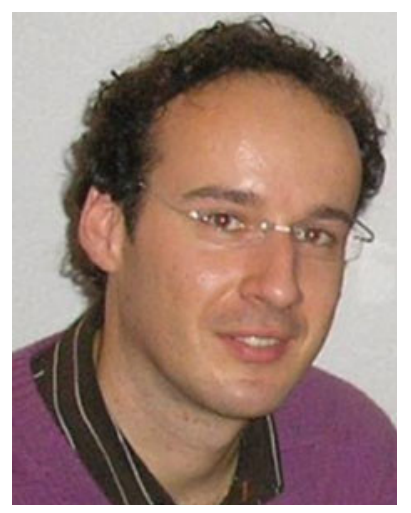

Aleksandar Jovanovic received the Diploma of degree in Telecommunication engineering and the MSc. Degree in Communication Systems from the Faculty of Electronic Engineering, Serbia, and Ecole Polytechnique Fédérale de Lausanne (EPFL), respectively. In September 2008, he started working toward the $\mathrm{PhD}$ degree in the area of global navigation satellite systems (GNSS) in the ESPLAB (Electronics and Signal Processing Laboratory) at EPFL.

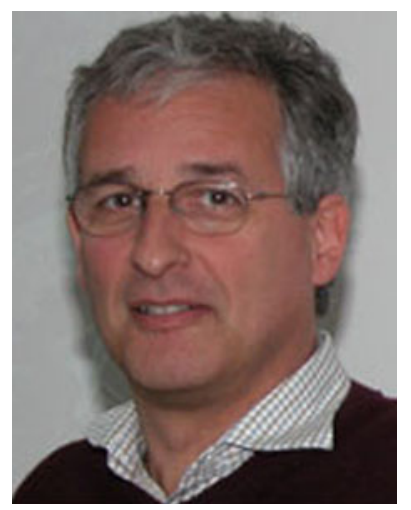

Pierre-Andre Farine is professor in Microtechnology at EPFLEcole Polytechnique Fédérale de Lausanne, Switzerland and is head of the Electronics and Signal Processing Laboratory (ESPLAB) at the Institute of Microtechnology IMT of the EPFL. 